\title{
LONG PATH TOWARDS TO SUCCESS IN ELECTROSPUN NANOFIBER YARN PRODUCTION SINCE 1930's: A CRITICAL REVIEW
}

\author{
Fatma Göktepe ${ }^{1}$, Beyza Buzol Mülayim² \\ ${ }^{1}$ Namık Kemal University, Çorlu Engineering Faculty, Tekirdağ, Turkey \\ ${ }^{2}$ Kırklareli University, Technical Sciences and Vocational School, Kırklareli, Turkey \\ Correspondence to: Fatma Göktepe E-mail: fgoktepe@nku.edu.tr
}

\begin{abstract}
:
Direct conversion of nanofibers into a yarn formed by electrospinning has begun to draw attention recently while pioneering attempts in fact go back to 1930s. Once nanofiber yarns are spun successfully by electrospinning, obviously, this would open new gates for many different applications. However, this is still a challenging task and there is no system accepted universally yet. There are more than 20 different approaches available so far but with serious limitations. In this review, they were categorized as (i) systems for production of parallel bundle of nanofibers and (ii) systems for production of twisted nanofiber yarns, presenting potential applicability of each with a critical point of view. The results show that some of the attempts mainly present basic conceptual ideas only. There are some works to produce real twisted nanofiber yarns continuously while mainly funnel, disc, or ring collectors have been used as the twisting element. However, there is limited information regarding stability of spinning system or control of yarn properties. This review also analyses the technical properties of electrospun nanofiber yarns summarizing the available data in terms of yarn properties such as fiber fineness, twist, production speed, mechanical properties, polymer types, and other important parameters available.
\end{abstract}

\section{Keywords:}

Nanofiber Yarn Production by Electrospinning, Twisted Nanofiber Yarns, Parallel Bundle of Nanofibers

\section{Introduction}

As well known, electrospinning is mainly a nanofibrous surface production method producing fibers with a fineness generally ranging between 50 and $500 \mathrm{~nm}$ providing a wide range of application areas from high-performance filters to hydrophilic textiles or from fiber-reinforced composites to biomedical textiles, electromagnetic protection, photovoltaic appliances, and so on. Its simple mechanism, easy modification on process parameters, and use of wide array of polymers have been the main advantage that lead to intensive works in electrospinning especially during the past decades.

Recently, however, there is also an interest on the production of uniaxial fiber bundles or twisted nanofiber yarns by electrospinning as well, while the first attempts in fact go back to 1930s. Direct conversion of nanofibers into yarn form would allow the use of nanofibers in traditional textile structures such as weaving, knitting or embroidery opening new gates for a greater number of applications. The combination of textiles and nanofiber-based yarns may also lead to new opportunities in smart and electronic textile fields [1]. For example, super-strong carbon nanofibers can be produced by using high-quality PAN nanofibers [2] or yarns composed of metal oxide nanofibers that display semiconducting behavior can be fabricated [3]. On the other hand, a recently introduced nanofiber yarn, NanoFront ${ }^{\mathrm{TM}}$ of Teijin, produced by sea-island method having an average fiber diameter of $700 \mathrm{~nm}$, for example, also shows the potential of such products in our life. Therefore, this work reviews the available methods for nanofiber yarn production by electrospinning summarizing yarn and fiber properties so that future potential of the novel approaches can be explored further. It is worth noting here that the scope of this review is electrospun yarn production systems only; therefore, wellknown literature on electrospinning field in general, is not repeated here.

Recent reviews on electrospun yarn production [4, 5] show the continuous interest in this field. However, a more comprehensive work is needed, and therefore, this review explores and discusses every single available study in detail with a critical point of view including pioneering patent works for electrospun yarn production back in 1930s as well. This work also indicates main strength and weakness of each work and compares overall properties of yarns (fiber fineness, yarn fineness, yarn twist, mechanical properties, etc.) in a systematic way.

Regarding yarn production systems by electrospinning, we divide more than 20 different approaches that are available into two main categories: (i) systems for production of parallel bundle of fibers and (ii) systems for production of twisted continuous yarns (Fig. 1). In fact, it is clear that the works by Formhals pioneer not only in electrospinning field but also in electrospun yarn production as well as by seven different patents dating between 1934 and 1944. Four patents by Formhals belong to parallel fiber bundle production [6-9], while other three patents are for twisted yarn production by electrospinning [10-12]. 


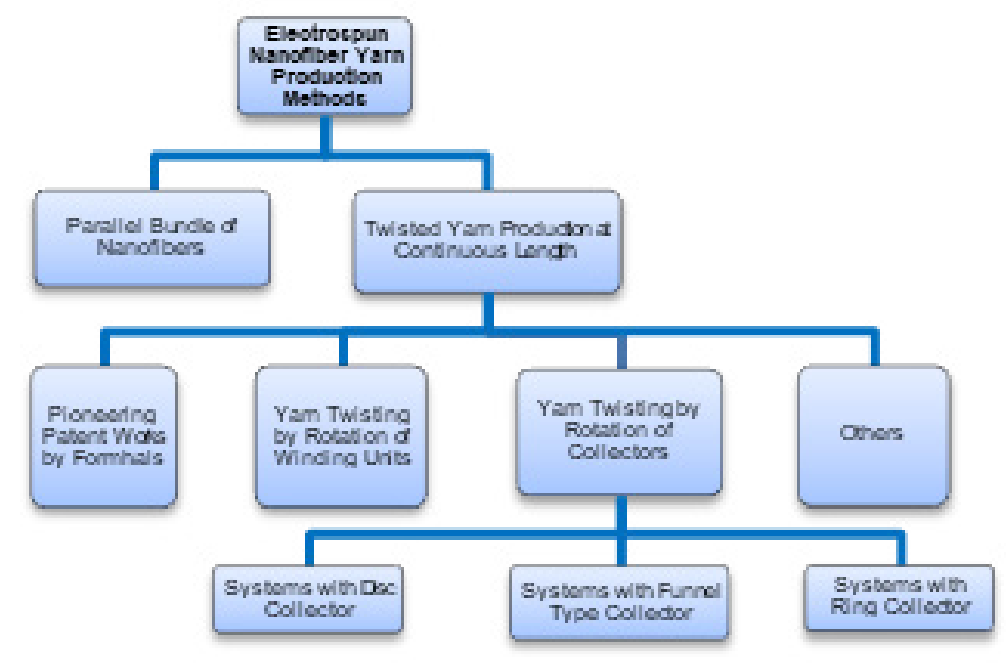

Figure 1. Classification of electrospun nanofiber yarn production methods in general

\section{Production Of Parallel Nanofiber Bundles}

In fact, parallel bundle of nanofibers cannot be considered as real yarns because there is no fiber twisting. Also, these approaches result in yarn-like fibrous short segments rather than providing a continuous yarn production. However, they might be considered as the first step in electrospun nanofiber yarn production, thus covered in this work.

The available approaches and methods are summarized in Table 1. The first method belongs to Formhals in 1938 [6]. In one of the conceptual designs by Formhals (Table 1a), it is reported that the length of artificial fibers can be controlled by means of the period of applied voltage, as applied voltage could be arranged intermittently in this system. If the applied voltage period is long, the length of fiber bundle would be longer. In his other designs, the system consists of polymer (11), power supply (19), non-conductive belt (21), electrodes (23), and positively loaded wire (25) (Table $1 \mathrm{~b}$ ). The same patent presents another approach (Table 1c) that uses a positively loaded trolley arm (87) and includes an extra grounding mechanism to change positive and negative loads. His next work in 1939 (Table 1d) is in fact a further improvement by adding a moveable prongs for fiber directing between collector and feeding system [8]. By using these prongs, the distance between nozzles and collector can be increased to gain more time for drying and better fiber orientation could be obtained. The next patent shows a system in which polymer is directed by air flow (Table 1e). A second design by the same patent presents nozzles that are in a circular arrangement while the polymers are directed towards the collector by an air jet [9].

Long after following the above mentioned pioneering works, we begin to see other attempts in this field. One of the first works belong to Pan et al. in 2006, producing parallel fiber bundles by a conjugate system leading fibers as fine as 346 $\mathrm{nm}$ (Table 1f) [13]. Li et al. used similar approach in 2007; this time composite yarns of PLLA/n-TCP were produced by multiple conjugate system [14]. The yarn tensile strength is reported as $0.31 \mathrm{cN} \mathrm{dtex}{ }^{-1}$, which is in fact quite weak. As the polymer concentration is increased to more than $5 \%$, stronger and thicker fibers are reported (Table $1 \mathrm{~g}$ ).

Another different approach is wet spinning (Table $1 \mathrm{~h}$ ), in which the voltage is applied to the electrode underneath the jet and water bath and then fibers are drawn by a winding roller [1]. Teo et al. also produced nanofiber bundles without any twist [15] by using a similar approach, indicating an increase in the average yarn diameter as the polymer feed rate and polymer concentration increase (Table $1 \mathrm{j})$.

On the other hand, the production of longer nanofiber yarn, more than a meter, is also reported by using a set up of a needle and a flat electrode connected to a high-voltage AC system, although there are no data available for fiber and yarn properties except fineness [16]. It is indicated that spinning behavior under an AC field is significantly different when compared to DC field because a visible yarn is seen emerging downstream from the needle instead of the mesh-like morphology observed when $\mathrm{DC}$ is used (Table 1k).

In another approach given by Mondal et al., electrospun nanofiber yarn was allowed to self-assemble within the spinning path; this process is referred as "e-spinning" and product as "e-spun yarn" [17]. The assembly of the split nanojets was initiated by an insulated rod placed between the top and counter electrode. This work also shows an increase in fiber fineness as PAN polymer concentration increases, as expected (Table $1 \mathrm{~m}$ ).

In this part, a series of works was reported by Lee et al. using multiple collectors in which the collectors were placed in different positions or different collector types were used [18]. In one design, for example, a metal planar collector (104a) was negatively charged and the second planar collector (104b) was neutral while the injector (103) was positively charged (Table 1n). They also used two planar collectors and a nonconductive component so that polymer was collected onto two collectors (Table 10), with fiber fineness of 1.4-2.5 $\mu \mathrm{m}$. 
Table 1. Different approaches for the production of parallel fiber bundles by electrospinning

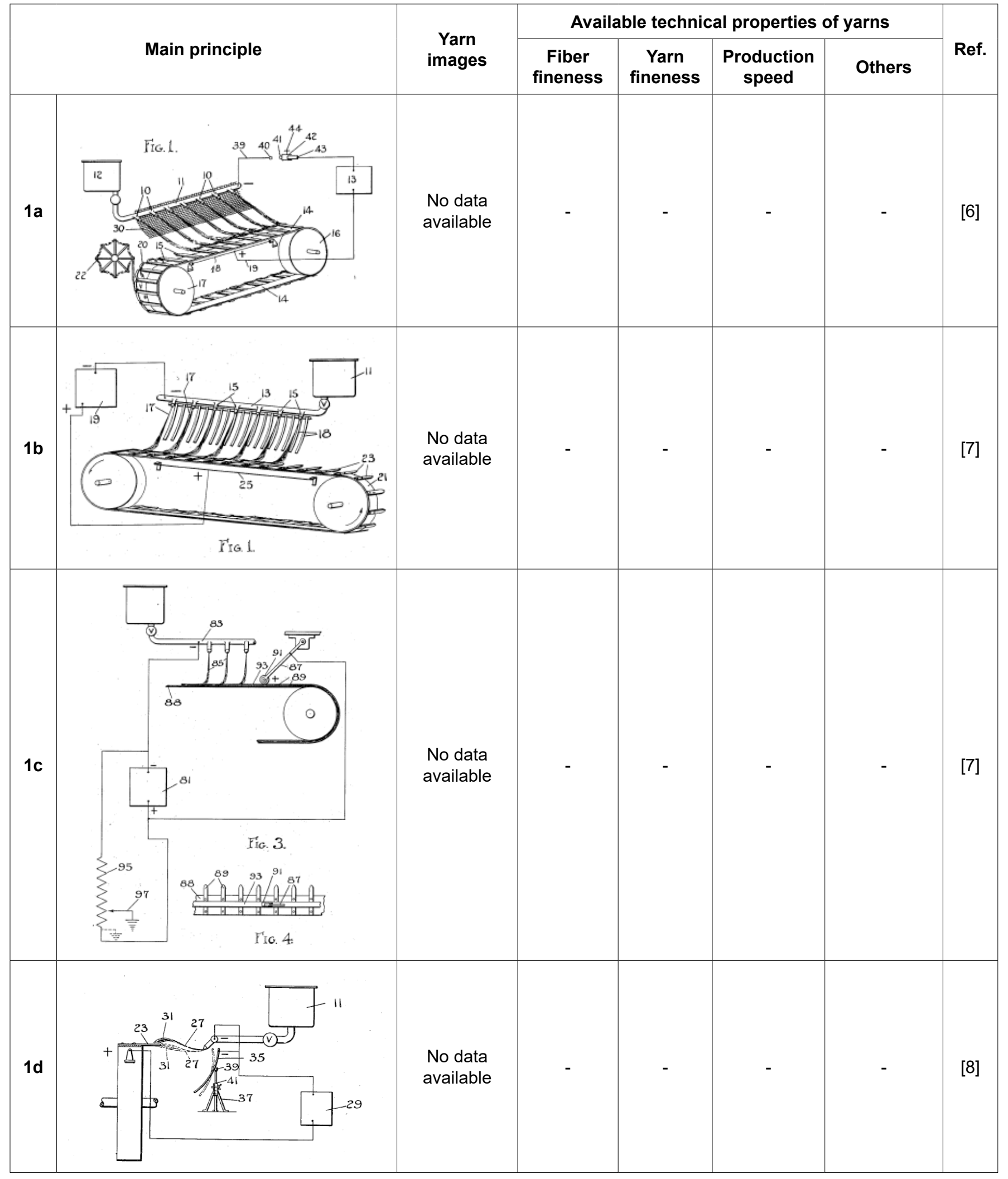




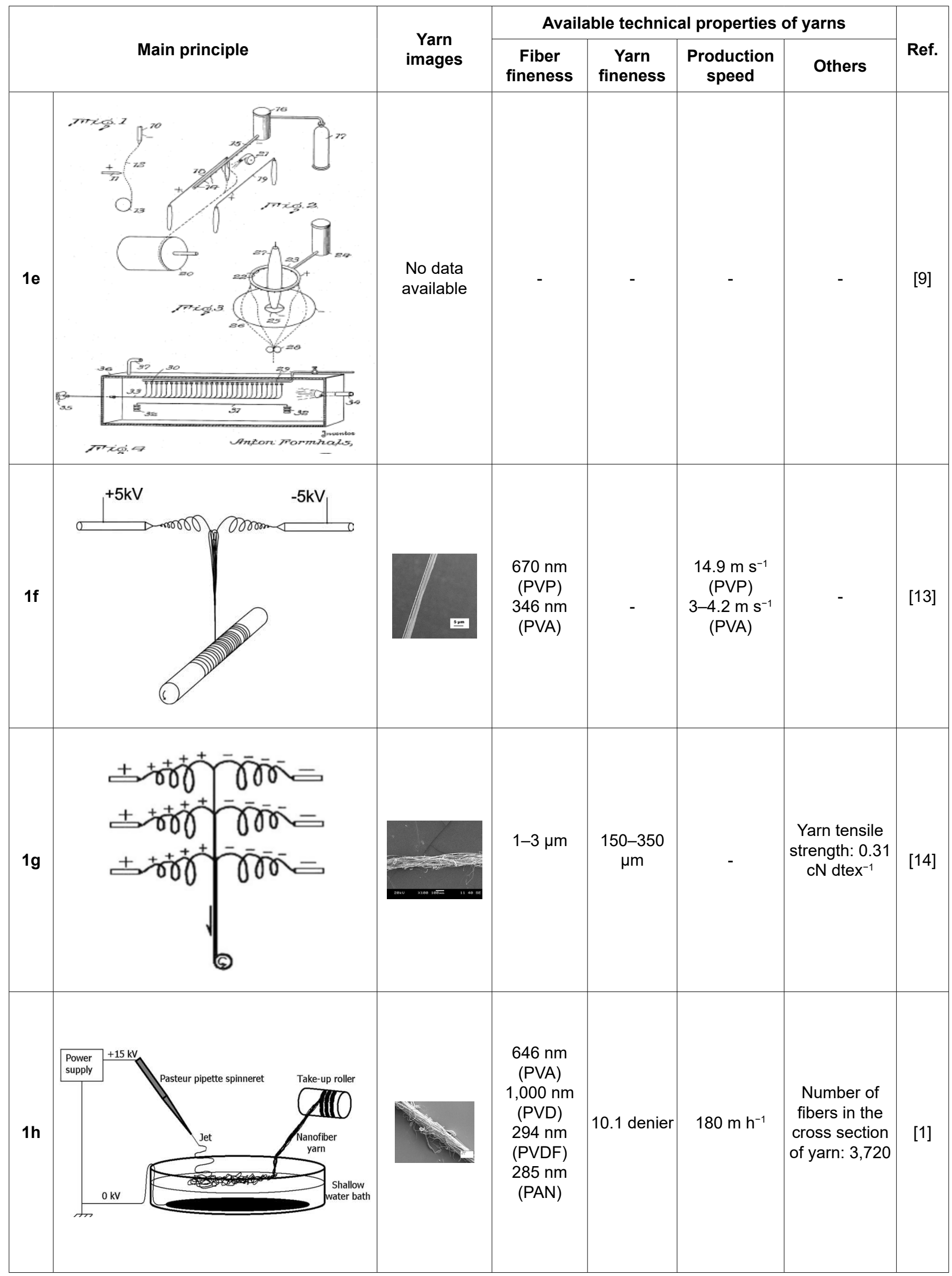




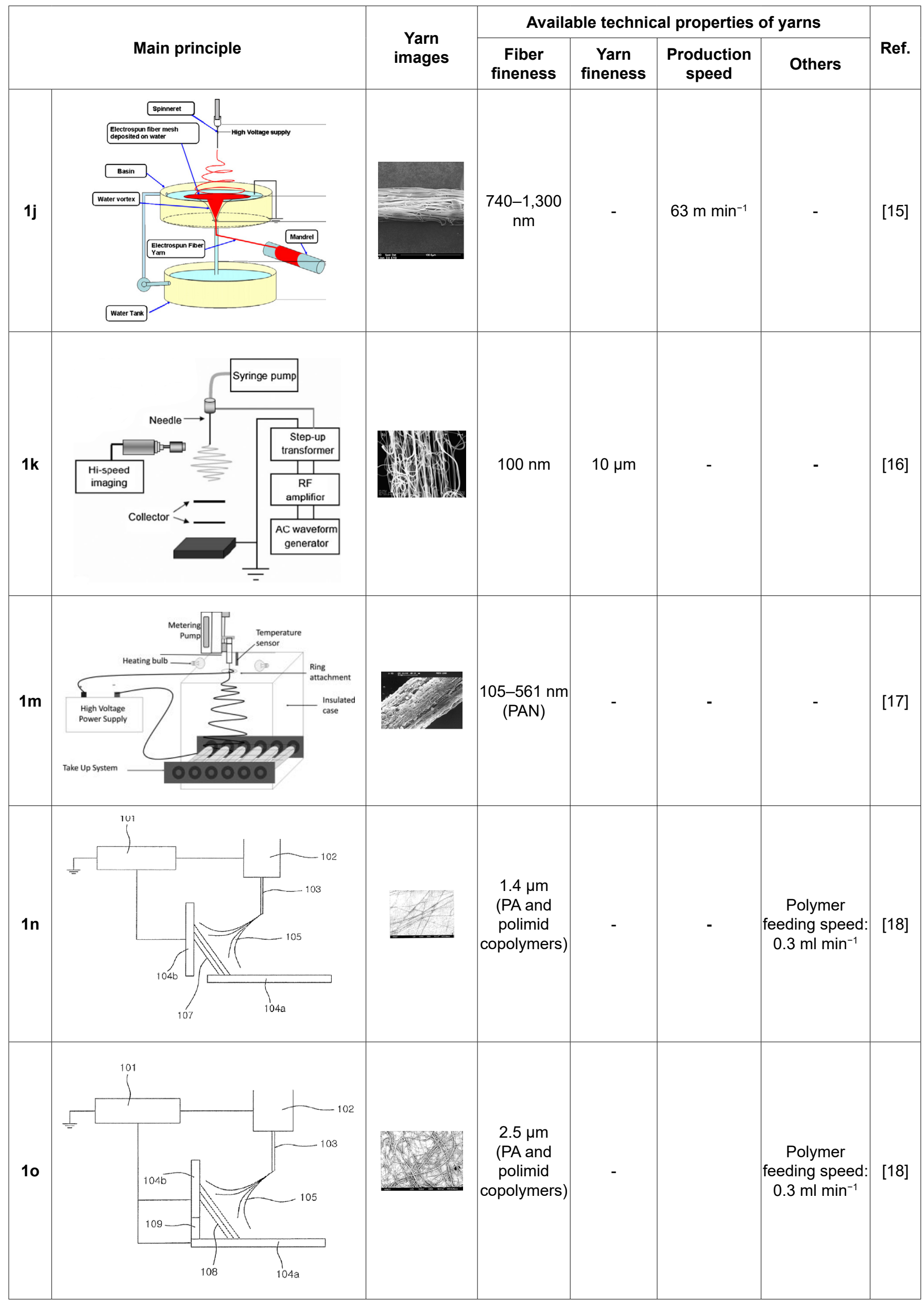




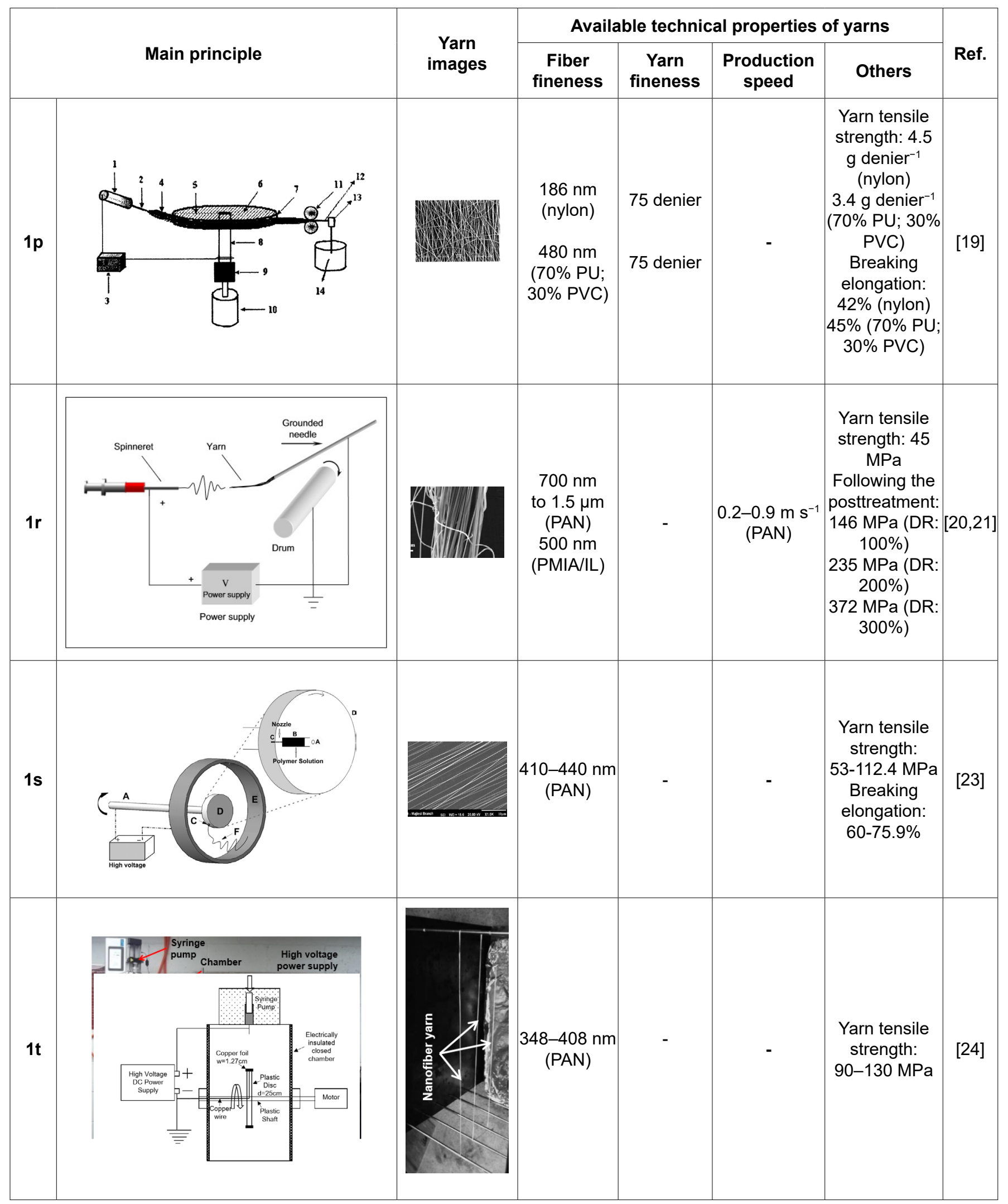

A disc-shaped conductive multilayer collector was also used [19]. By using nylon polymer, quite fine fiber, for example, 186 $\mathrm{nm}$ in diameter, was reported, while yarn tenacities by different polymer types were also investigated (Table 1p).

In another work, a grounded needle tip was used to induce a self-bundling of nanofibers at the beginning of electrospinning process and then winding fibers onto a grounded rotating collector [20]. A difficulty in yarn production by PHBV polymer was reported because of low conductivity $\left(0.6 \mu \mathrm{S} \mathrm{cm} \mathrm{cm}^{-1}\right)$; however, this difficulty was overcome by using organic salt. The yarn production speed was also increased to $0.9 \mathrm{~m} \mathrm{~s}^{-1}$ by using organic salt with PAN polymer, while the average diameter of single fiber was decreased because of the 
increased charge density in jets and thus imposing stronger elongation force (Table 1r). The same research group used the same self-bundling electrospinning technique combined with posttreatments such as drawing and annealing similar to conventional production. Following an enhanced alignment, a higher degree of crystallinity and higher molecular orientation after posttreatments as well as an improvement in mechanical performance was obtained by approaching the strength value of corresponding conventional fibers [21]. Here, different polymers were also used, while the yarns produced with PAN polymer looked fluffy similar to the work of Xie [22].

Dabirian et al. used a different approach, namely, electrocentrifugal, and compared it with electrospinning. They concluded that it was a powerful technique in low viscosity polymer use and more suitable for the production of aligned nanofibers compared to electrospinning because bending instability of jets could be reduced via centrifugal force through the effect of surrounding air contacts (Table 1s) [23]. A revolving disc collector was also used for such a production in which the edge of the disc was copper while inner part was plastic so that fibers could be collected on the edge part only [24]. The electrospun nanofibers were peeled off from the copper foil in a kind of sliver form after immerging in distilled water and then mounted on a drying rack to keep them in tension and dried at $95^{\circ} \mathrm{C}$ for $5 \mathrm{~h}$. (Table $1 \mathrm{t}$ ). The collector speed was reported as $600 \mathrm{rpm}$ for optimum fiber orientation, and the tensile strengths were also analyzed.

The different approaches regarding parallel bundle of fiber production by electrospinning that are summarized earlier can be considered mainly as the initial works that can lead to twisted electrospun nanoyarn production. Therefore, they are considered as valuable attempts for successful twisted electrospun yarn production.

\section{PRODUCTION OF TWISTED NANOFIBER YARNS BY ELECTROSPINNING}

As mentioned earlier, the main challenging issue in electrospun yarn production has been the continuous production of twisted yarn spinning. Once such a universally acceptable successful system is developed first, the next step will be the control of yarn parameters during spinning. Naturally, the available works have been mainly dealing with the first step for a start: a successful continuous nanofiber yarn spinning with acceptable yarn properties. We classified the different approaches used for such an aim into four different groups and are summarized in the following text.

\subsection{Pioneering Works by Formhals}

The pioneering works for such an aim also belongs to a serious of patent works by Formhals as the first one was dated as 1934 [10] presenting a positively charged serrated wheel (f) in a polymer vessel while the metal disc $(\mathrm{g})$ works as collector. Following washing ( $\mathrm{h}$ and $\mathrm{I}$ ), drying $(\mathrm{m})$, and drawing process, yarn twisting was described as passing through an "O" point, although that was not shown in available drawings (Table 2a).
There is no data for yarn twist level or structure, either.

Formhals also described core-spun yarn production by electrospinning [11] (Table 2b). On the other hand, a rotating collector was used to collect fibers by his next patent in 1943 [12]. Here, following a high electrical field produced between two electrodes, a transmutation of the liquid evolved from nozzles into fibers takes place in this field and is attracted by the counter electrode (Table 2c). Then, fibers in the proximity of the counter electrode get into a state of equilibrium or suspension, and in this neutral zone, the fibers consolidate into a fiber band and it is drawn out of the electrical field. Finally, yarn twist was applied by a rotating collector similar to a funnel or rotor (17). There was no data for technical parameters such as production speed, yarn fineness, yarn twist, or fiber fineness; however, one would not expect these in those years, as it mainly presented conceptual idea pioneering today's many attempts in this field. This concept also seems to be the inspiration for some works even today, for example, the systems developed by Xie [22] or Ali et al. [25].

\subsection{Twisted Electrospun Nanofiber Yarn Production Methods by Rotation of Winding Units}

One of the first attempts in this part belongs to Dabirian et al. in 2007. In their design, a positively charged injector and a negatively charged planar collector were used, and a negatively charged bar was added to manipulate electric field while take-up unit is placed onto a revolving platform to apply twist (Table 3a) [26].

Another approach here is a conjugate system (Table 3b) [27]. This kind of yarn was reported for many potential applications, such as preparation of carbon nanofiber yarns or supports of cell culture. A similar approach was also given by Hajiani et al., but it includes a neutral cylinder (Table 3c) [28]. They indicated that twist angle of nanofibers increased while yarn diameter decreased by increasing twist rate. Results also showed that stress-strain values of yarn could be improved considerably by increasing twist rate attributing this to the influence of spinning triangle. On the same system, PLLA yarns were also produced to investigate the effect of different solvents, indicating that solvent type affected fiber fineness (Table 3c) [29]. The effect of processing parameters on morphology, diameter, and mechanical properties of PLLA yarns was also analyzed by the same research group, revealing that yarn diameter mainly depended on take-up rate. Experimental data on strength and modulus of the yarns also indicated that polymer concentration, voltage, and a combination of these parameters have been the most significant factors affecting the mechanical properties [30]. $\mathrm{TiO}_{2}$ nanofiber yarn was also produced by the same researchers for potential applications such as photo catalysis and sensors in electronics [31].

In another approach, the fibrous strips are produced first and then these are converted into yarn by plying [32]. In this approach, both spinneret and the auxiliary electrode were connected to the cathode of high power supply, while the anode was inserted into the bottom of the bath (Table 3d). Upon application of high voltage, PA6 fibers were obtained on the bath surface and 
Table 2. The pioneering works by Formhals for twisted electrospun yarn production

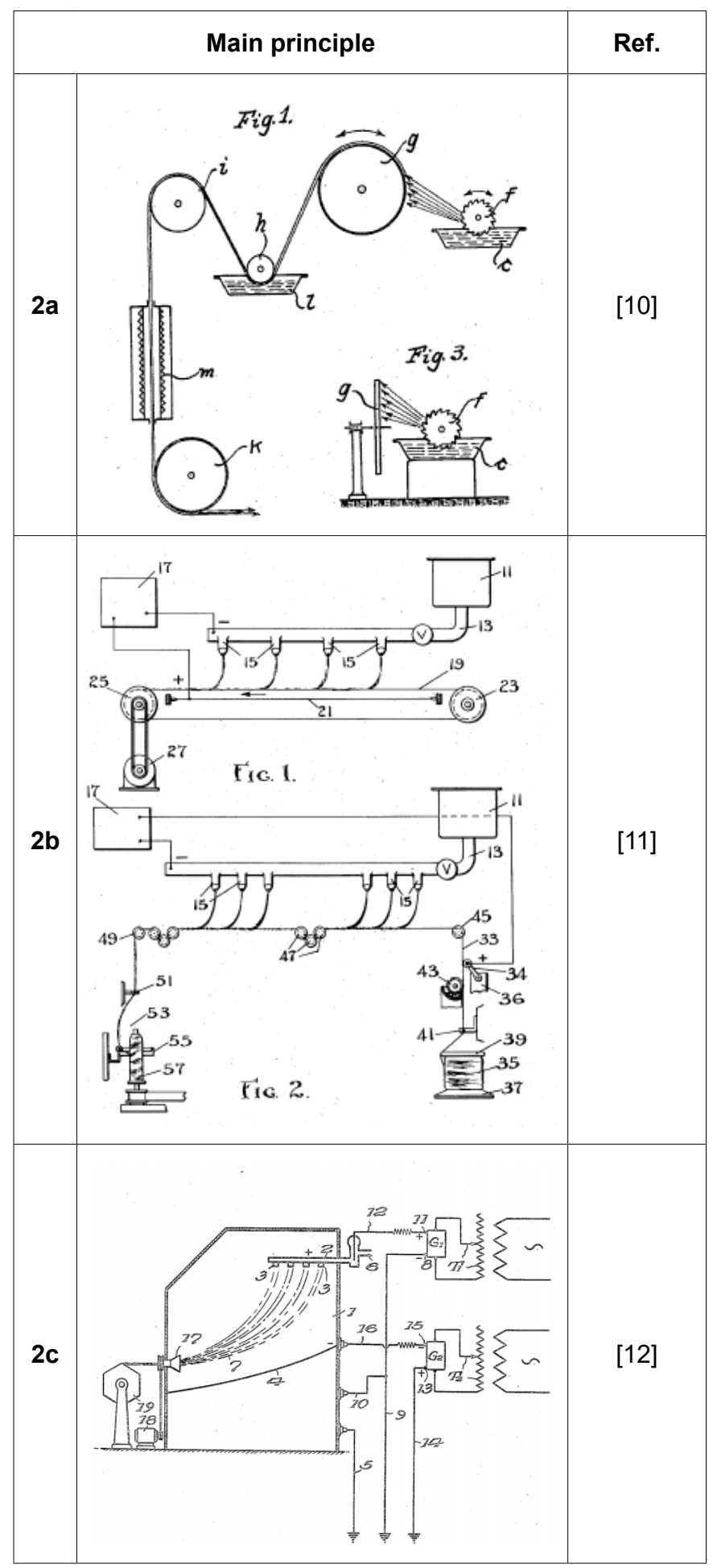

yarn-like structures were obtained by collecting these and a heating process was applied. The second part of the system had a guide roller and filament arrangement set-up to produce the so-called filament yarn. As a final step, a vacuum steaming process was applied to fix the yarn twist. However, provided yarn image does not have a uniform structure having plying marks on yarn surface. Also, it is difficult to mention about real filament or nanofiber yarn spinning here because the process has been discontinuous.

\subsection{Twisted Electrospun Nanofiber Yarn Production Methods by Using Rotating Collectors}

In this part, the available systems can be classified into three different groups, as shown in Fig. 1, depending on the form of rotating collector being a disc or a ring or a hollow hemispherical-shaped rotor or funnel.

\section{a) Systems with Disc Collector}

The first work in this group used two discs: one for twisting and the other for winding (Table 4a) [33]. The same research group also produced core-spun yarns aiming for industrial and medical applications (Table 4b) [34]. Similar approach was also reported by Lee et al. by a series of designs (Table 4c-4f) [18]. However only fiber images were provided.

A rotating disc and conjugate nozzles have also been used [35]. The most significant part here was including a heating zone so that yarn was passed through this zone before winding, as heating the PAN fibers under tension was reported to increase initial modulus and stress (Table 4g). A similar approach was also used by Wu et al. [36,37]. They used two injectors, a neutral metal disc (NMD), a neutral hollow metal rod, and a winding unit to produce continuous twisted yarns of PAN (Table $4 \mathrm{~h}$ ). The effect of the main parameters on yarn and fiber properties were studied extensively here, reporting that at higher voltages, the fibers were scattered around, indicating that the fiber formation at low polymer feed rate was very few but polymer droplets reach toward the collector spoiling the conical fiber formation zone at higher rate. Also, both fibers and yarns become coarser as polymer feed rate was increased. They also investigated the effect of distance between injectors and reported that as the distance increases, fiber and yarn become coarser. The rotation speed of NMD was also studied, indicating that fiber orientation becomes inferior at higher speed relating this to airflow perturbation at high NMD speed. On the other hand, they reported that it has no significant effect on fiber fineness but yarns became finer.

A different method dealt with the production of a fiber sheet first around a rotating disc and then twisting these by using a motorized spindle up to a twist level of $1300 \mathrm{t} \mathrm{m}^{-1}$ (Table 4j) [38]. Following this, carbonization process was applied. However, there was no real yarn twisting or spinning process here, as the production was discontinuous. Also there was no further data provided for produced yarns.

A grounded revolving wheel was also used to obtain a better orientation and stronger yarn, as a unidirectional tow of electrospun nanofibers were linked together and then twisted using an electric spinner (Table 4k), obviously not being a real yarn spinning system [39].

\section{b) Systems with Ring Collector}

This is relatively a new approach that is reported more recently in 2015 [40]. Two injectors and a ring collector have been used in this system, probably inspired by conventional ring spinning (Table 5). The yarns having up to 4,000 tpm nominal twist level depending on the speed of the ring collector were produced. 


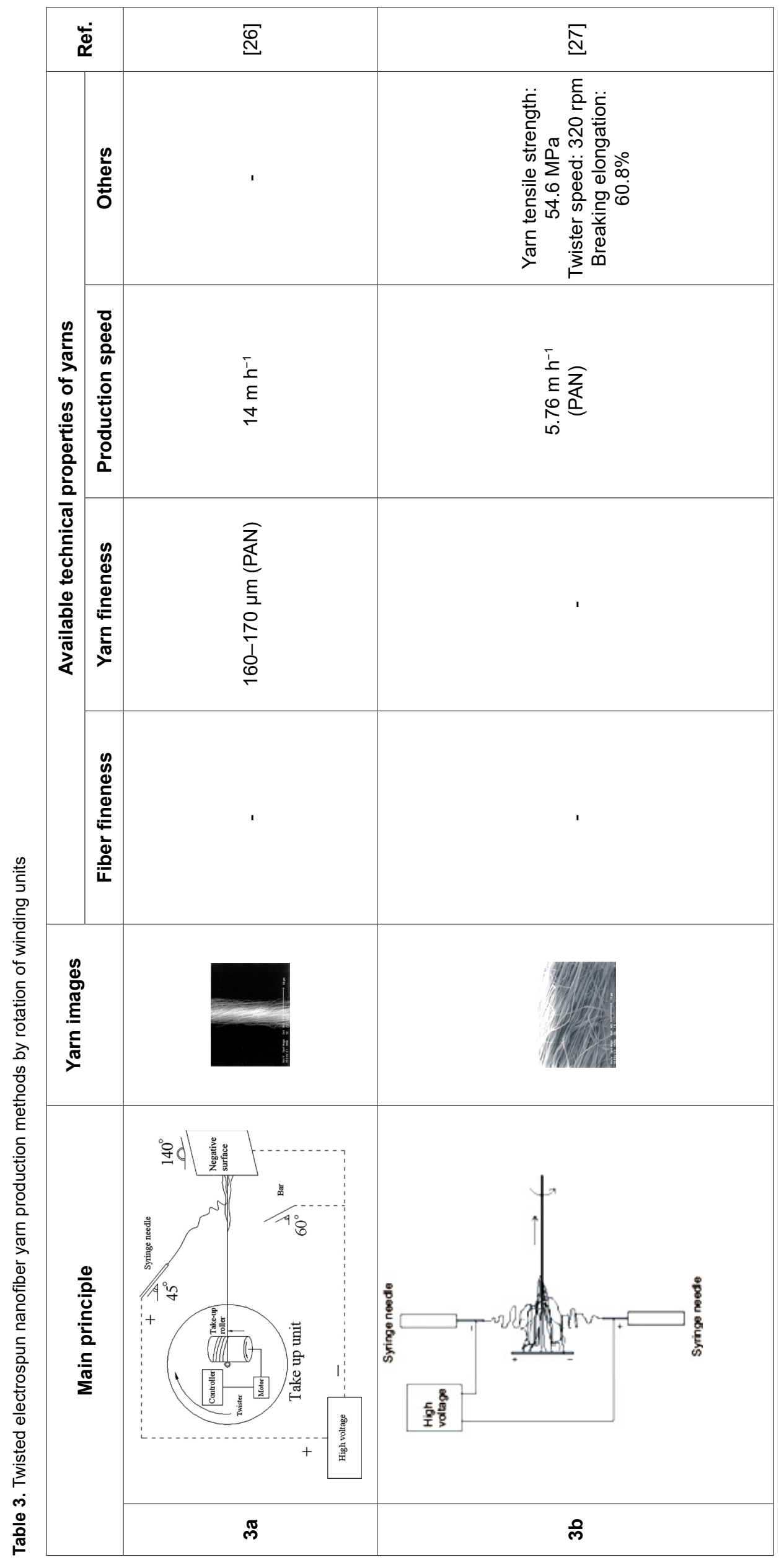




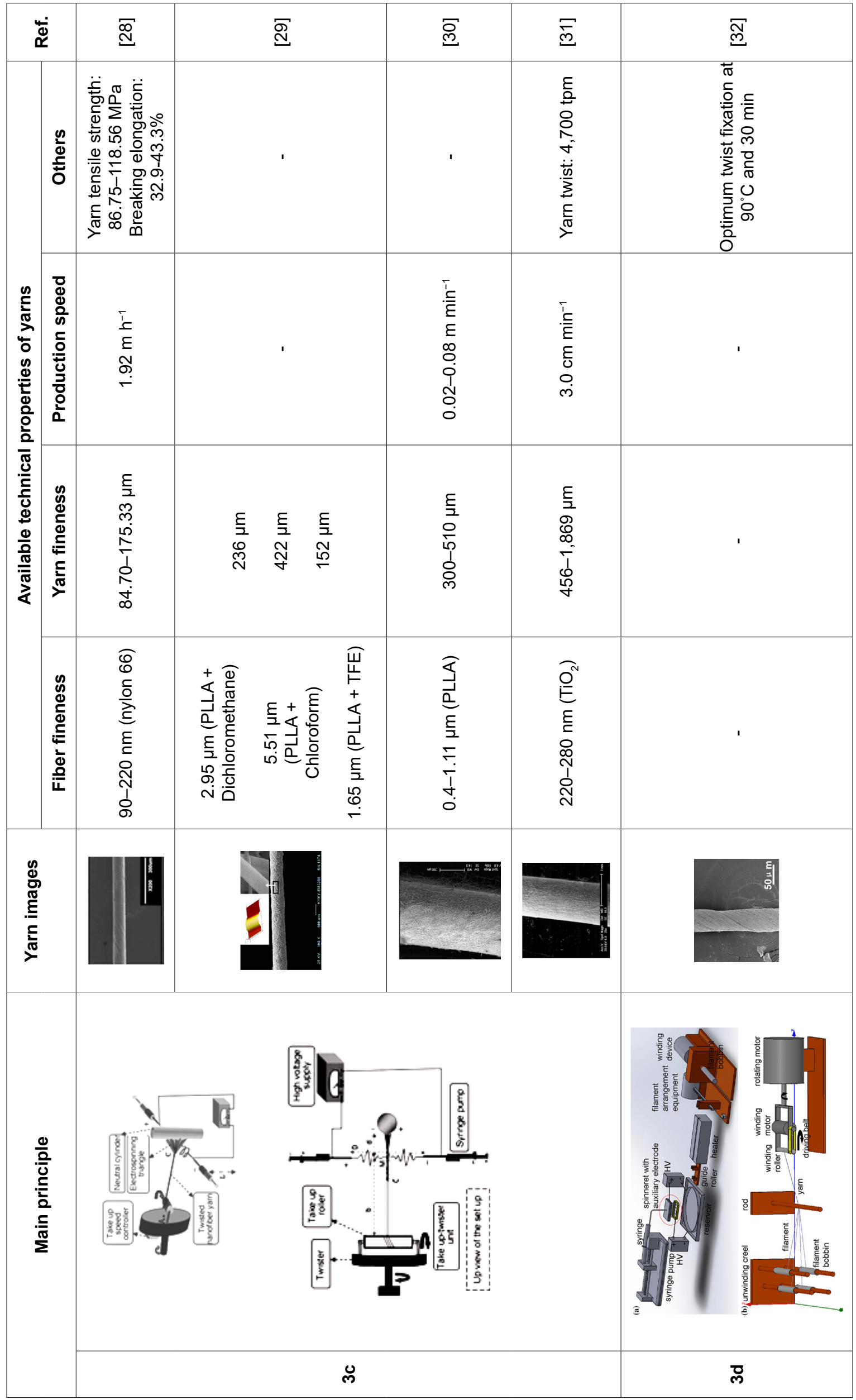




\section{c) Systems with Hollow Hemi-spherical Shaped Rotor or Funnel Type Collector}

This type of nanofiber yarn production method has been highly favored by different researchers working in this field, probably because of the inspiration by the earlier work of Formhals [12]. Some of the first examples use a hollow semispherical-shaped collector in a way similar to a rotor in conventional open-end rotor yarn spinning [3, 41,42] (Table 6a-6c). By Lotus [3], the hollow metal hemisphere was rotated between 100 and 1,000 rpm to apply twist to the ejected fibers. Another research group produced PLA nanofiber yarns by a similar approach that investigated the tensile properties of these yarns as well (Table 6b) [41]. On the other hand, there are systems in which both hollow hemisphere formed collector and yarn take-up unit have been rotating (Table $6 \mathrm{c}$ ) and very flexible fabric by PAN nanofiber yarns with a cover factor of 0.44 was produced [42].

Mainly being similar but using a funnel-shaped collector was also reported to produce PAN nanofiber yarns (Table 6d) [43]. The funnel-shaped collector consisted of two parts: the funnel and a vacuum pump as the air suction through the funnel is the main difference. In this work, the effect of distance between spinneret and collector as well as the effect of $\mathrm{LiCl}$ addition to the PAN polymer was studied, emphasizing the synergetic effect of both air flow and $\mathrm{LiCl}$ addition. However, there were no data available for yarn or fiber properties. Furthermore, there was no information on winding system either; therefore, probably, short length of yarn production has been the case here.

Use of funnel-shaped collector has been perhaps one of the most promising approaches because there have been other attempts as well (Table 6e, 6f) [25, 22]. Using the same principle, the influence of twist level on the tensile properties of nanofiber yarns was studied by Ali et al., indicating a peak level at 3,500 tpm and exhibiting a similar trend to conventional ring spun yarns [25]. However, yarn twist level was based on a calculation by using the relationship valid for conventional yarn production (Equation 1). It was also reported that the yarn and fiber fineness decreased while twist angle increased as the funnel speed and winding rate increased. Similarly, fiber and yarn fineness decreased as polymer concentration and polymer flow rate increased. The yarn production speed was between 0.33 and $5.0 \mathrm{~m} \mathrm{~min}^{-1}$. Different from this, Xie included a back disc electrode to control the fiber deposition [22]. In this so-called disc-aided electrospinning setup, the syringe needle was plugged into the disc hole with about $0.5 \mathrm{~cm}$ needle orifice off the disc surface. The high voltage was directly connected to the disc electrode, and the back disc electrode was in physical contact with the needle. At the same spinning distance, the deposition area for the disc-aided electrospinning was only one quarter of that conventional electrospinning, leading to higher areal fiber density at smaller deposition area.

$$
\text { Yarn twist }(t / m)=\frac{\text { Spindle speed }(\mathrm{rpm})}{\text { Yarn production speed }(\mathrm{m} / \mathrm{min})}
$$

As a further step to the works pointed earlier, four symmetrical nozzles were also used to feed polymer to a funnel-shaped rotating collector [44-46]. Rotation of the funnel collector was adjusted in the range of $0-180 \mathrm{rpm}$, and the take-up speed of yarn winder was $40 \mathrm{~cm} \mathrm{~min}{ }^{-1}$ while $18 \mathrm{kV}$ was given as optimum level. Similar to the work of Ali et al. [25], fibers became finer while yarns became coarser as the applied voltage increased. As winding rate increased, however, yarn twist level increased but yarns became finer. Strength and elongation of yarns increased from 1.81 (twistless) to $55.70 \mathrm{MPa}$ (375 tpm) and from $24.47 \%$ (twistless) to $41.31 \%$ (375 tpm), respectively (Table 6g) [44, 45], that is, yarns became stronger as the twist level increased. Although basic principle was similar, multinozzle bubble electrospinning apparatus including an air chamber was also introduced by same group to produce PAN nanofiber yarns (Table 6h) [46]. The stable nanofibers were obtained at $34 \mathrm{kV}, 1200 \mathrm{ml} \mathrm{min}^{-1}$ airflow, and $32 \mathrm{ml} \mathrm{h}^{-1}$ polymer feed rate for a uniform yarn production. They also reported that yarn twist increased depending on collector speed. Yarn tensile strength and elongation at break values also increased with yarn surface twist angle.

An interesting work was reported by Yang et al. [47] using a system similar to the one by Lotus et al. [3]. A novel scaffold with improved mechanical strength was fabricated composing both aligned nanoyarns and random nanofibers (Table 6k).

Similar to these works described earlier [22, 25, 44-46], Levitt et al. used funnel-shaped rotating collector as well to produce twisted nanofiber yarns but by using three different polymers, namely, PVDF-TrFe (65/35\%), PAN (15\%), and PCL (10\%), at funnel speeds of 500, 700, 900, and 1,100 rpm (Table 6m) [48]. As funnel speed was increased, the nanofiber yarns became finer when PAN and PCL were used, but, interestingly, they became coarser with PVDF-TrFe. As expected, yarn twist angle was increased as funnel speed was increased for each polymer type. PCL and PVDF-TrFe yarns had a significantly higher strain to failure than PAN yarns. Maximum tensile strength of $P C L$ and PVDF-TrFe nanoyarns was observed at funnel speed of $900 \mathrm{rpm}$, while this was at $700 \mathrm{rpm}$ for PAN yarns. Another interesting part of this work has been the analysis of nanofiber yarn porosity, as, apparently, it is greatly affected by polymer type rather than yarn twist angle. A rotating conical-shaped funnel collector was also used for the production of PAN-based twisted nanofiber yarns at continuous length. The average fiber diameters were reported as $500 \mathrm{~nm}$, as yarn diameters range between 72 and $100 \mu \mathrm{m}$ [49]. Use of conical funnel collector was also reported by Jin et al. more recently (Table 6n) [50], mainly investigating the effect of collector speed and polymer concentration as well as analyzing thermal resistance and wicking properties for polysulfone amide (PSA) electrospun yarns.

\subsection{Other Methods}

One example in this part presents a method similar to the texturizing process in conventional yarn production (Table 7a) [51]. In another work, hybrid yarns for artificial tendon/ligament scaffolds were produced by coating electrospun nanofibers on polyamide (PA) monofilaments first and then a number of nanofiber-coated monofilaments were twisted into yarns (Table 7b) [52]. 
Table 4. Twisted electrospun nanofiber yarn production methods that use rotating disc collectors

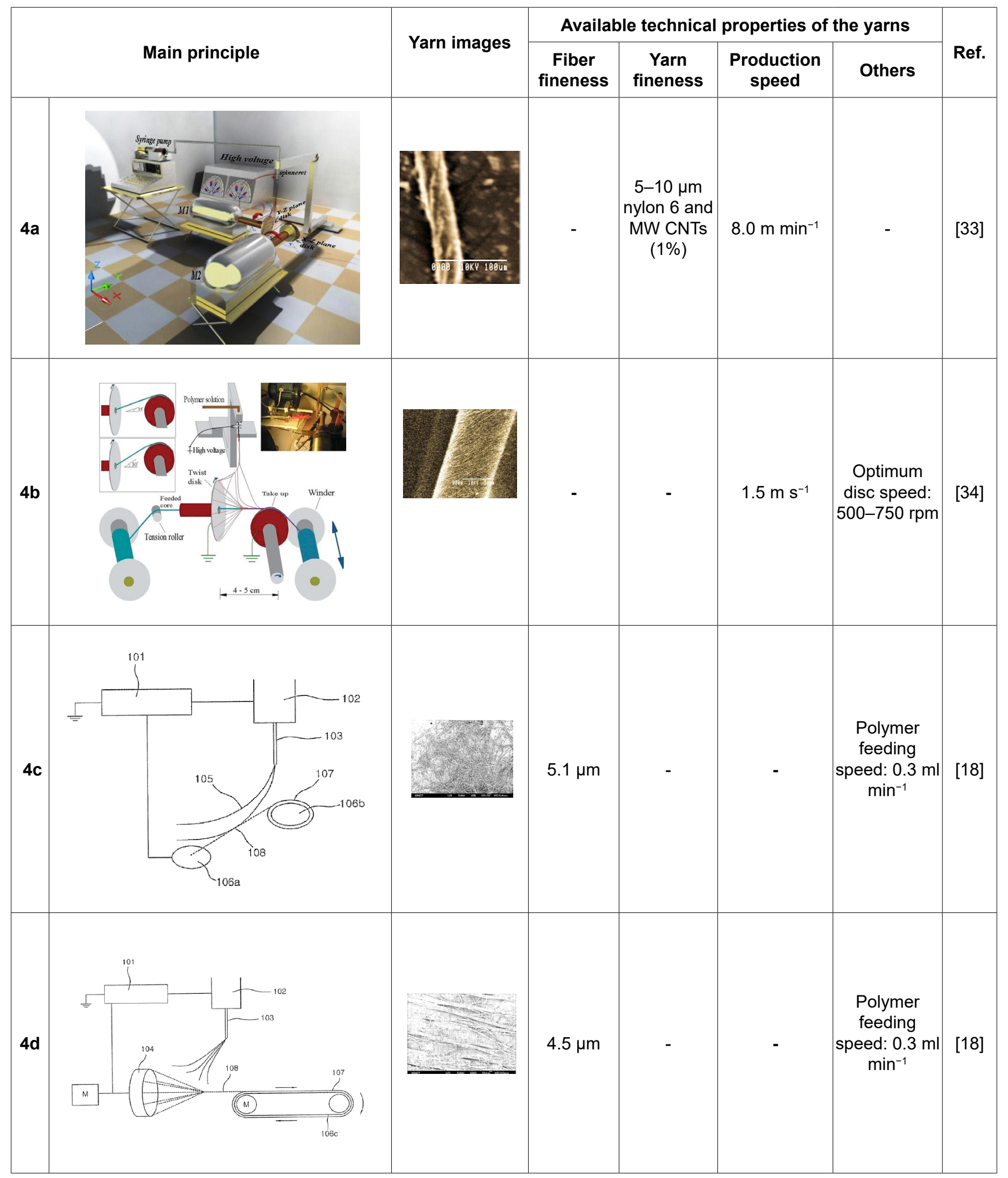




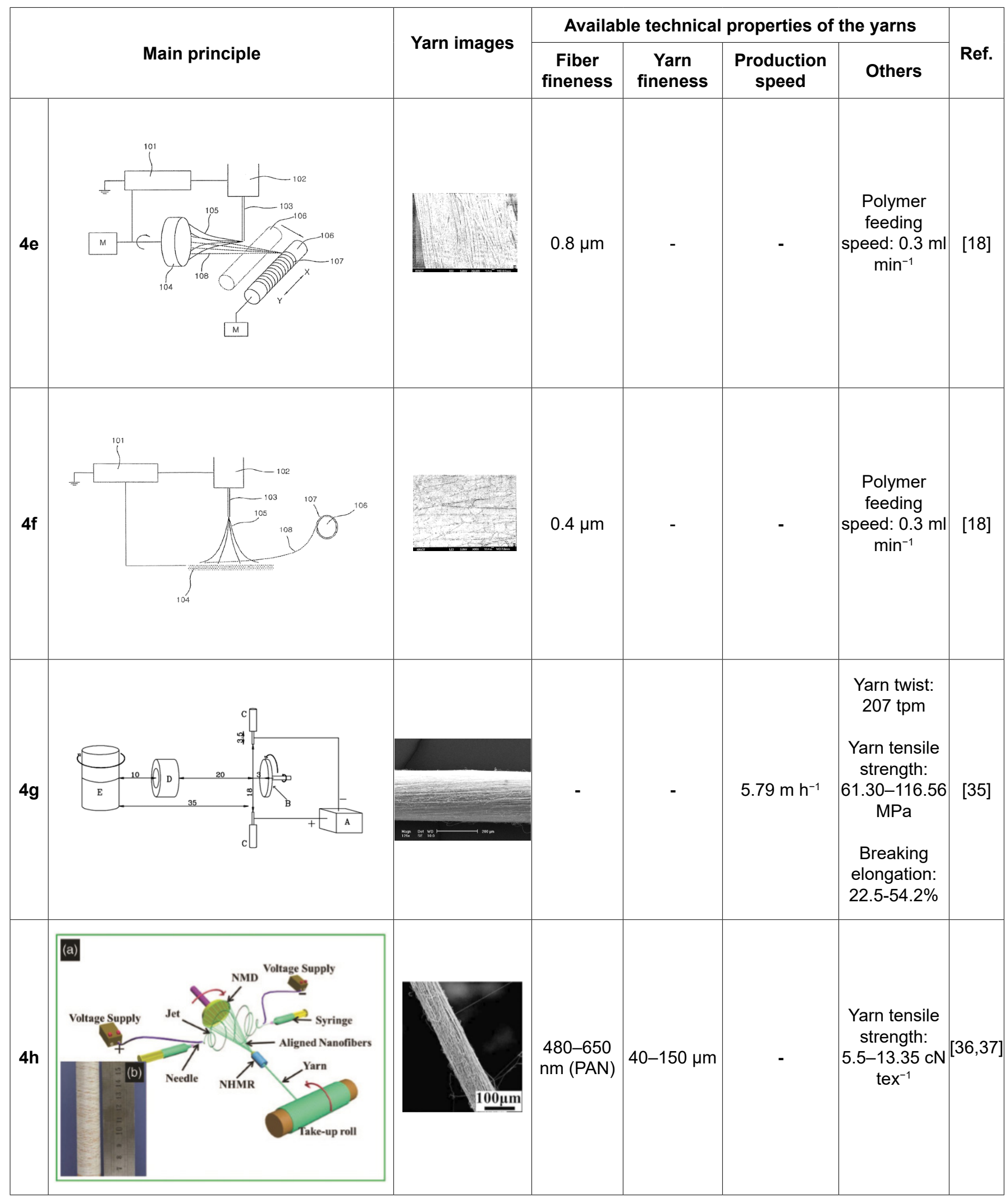




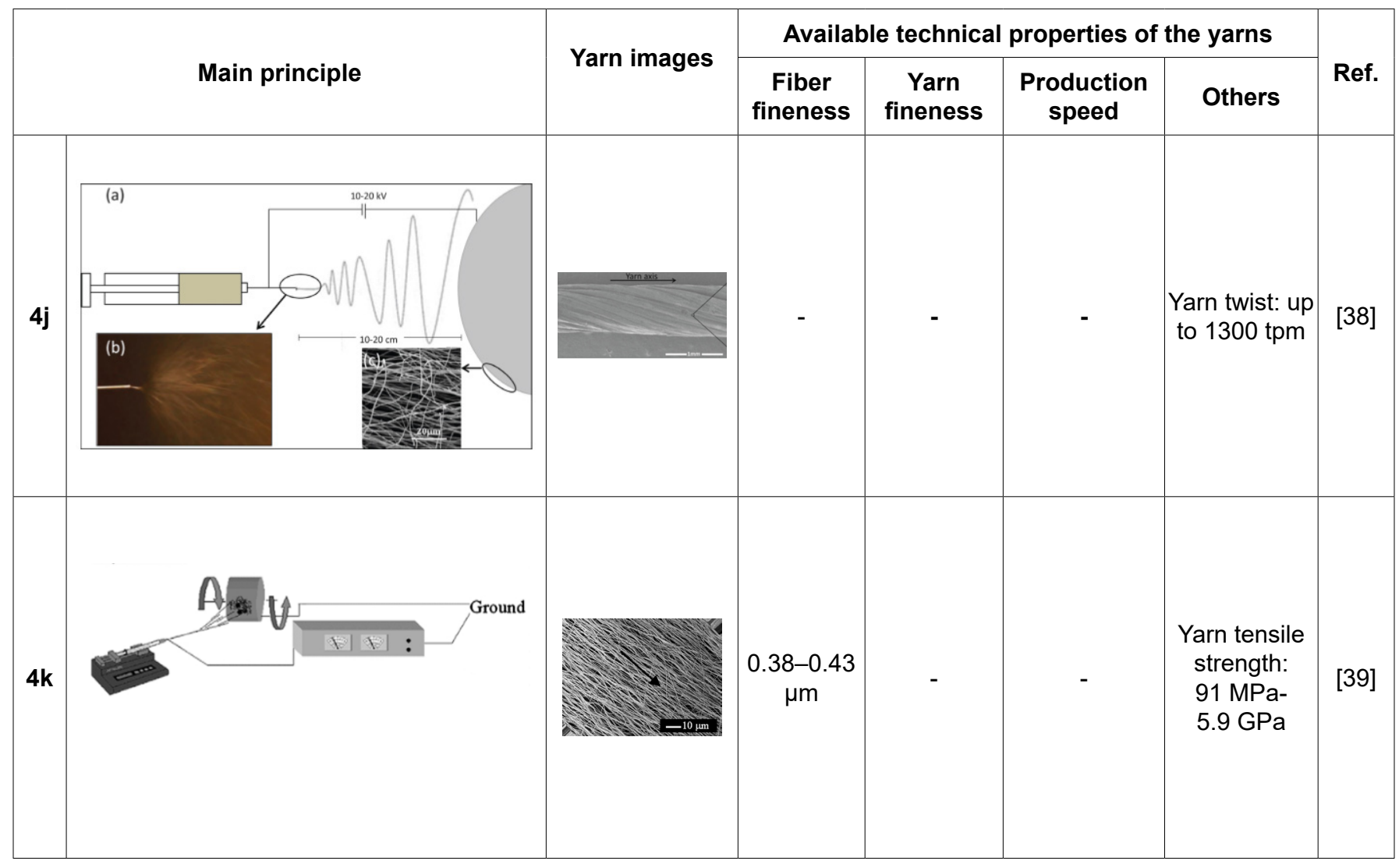

Table 5. Twisted electrospun nanofiber yarn production methods that use ring collector

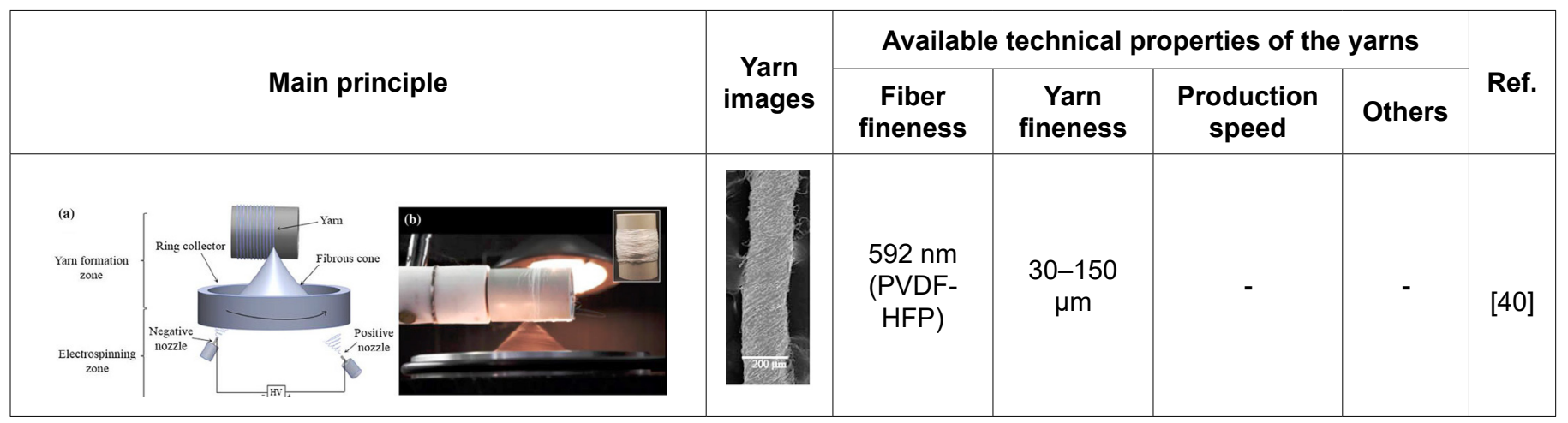

Interestingly, yarn twisting was also applied by air jet using a multinozzle system [1]. Here, a band or nonwoven surface was produced first and then these bands were twisted by an air jet to obtain a twisted yarn (Table 7c). Electrospinning of fibers onto a water bath with a vortex and drawing fibers through this vortex by applying some twist and then wound up into a yarn form might also be included in this part (Table $7 d$ and $7 e$ ) $[1,53,54]$. Similarly, the water bath was also used to collect fiber bundles using two injectors that have opposite charges, and fiber jets are collected onto a static water bath [55]. The produced yarn has been named as "nano open-end yarn" just like open-end yarn spinning in conventional textile yarn production (Table 7f). However, there was no further information about twisting mechanism, although authors referred to the twisted yarn production here.

There have been rather different approaches as well such as production of $1 \mathrm{~m}$ length of aligned electrospun nanofiber yarn by using two metallic twisting tubes that rotates at opposite directions positioned at a certain distance (Table 7g) [56]. Then twisted yarns can be wound onto a winding tube that is made of low dielectric material. In this work, they also attempted to define nanofiber modulus in nanofiber yarns by using theoretical relationship for conventional staple yarns given by Equation (2):

$$
\frac{E_{y a r n}}{E_{\text {fiber }}}=\cos ^{2} \propto(1-k \operatorname{cosec} \propto)
$$

where $E_{\text {fiber }}$ is the fiber modulus, $E_{\text {yarn }}$ is the yarn modulus, $\alpha$ is the yarn surface twist angle, and $k$ is a constant related to fiber length, coefficient of friction of fiber, fiber radius, and fiber migration period within the yarn [57].

On the other hand, a needle, two conical-tipped grounded copper rods, and a grounded knife-edge bar have been used to produce quite compact and regular staple nanoyarn (Table 7h) [58]. Here, nanoyarn was fabricated between copper rods as a bundle of fibers first and then twist was applied to this bundle by rotating copper rods in opposite directions. In this 
Table 6. Twisted electrospun nanofiber yarn production methods that use rotating hollow hemispherical-shaped rotor or funnel type collectors

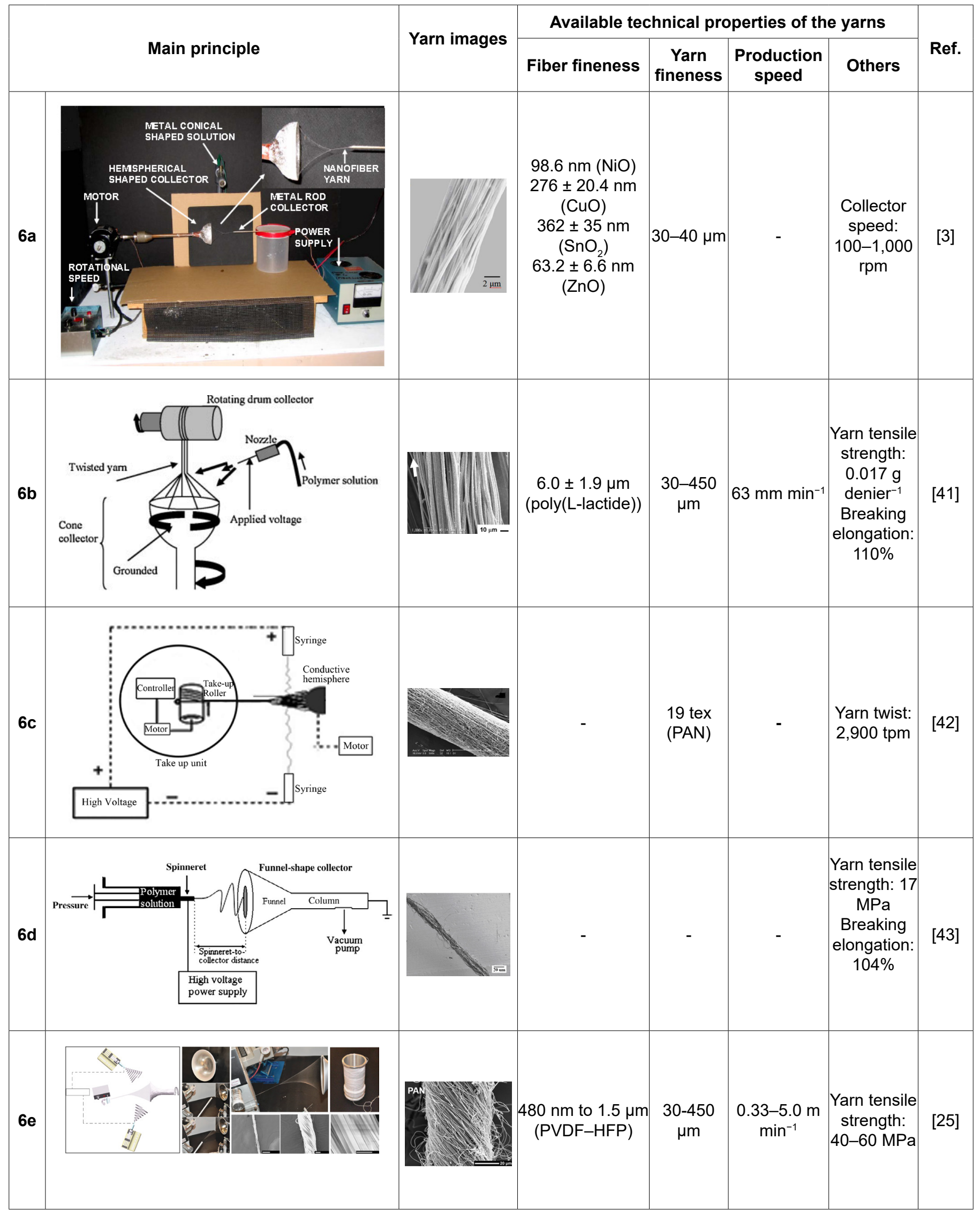




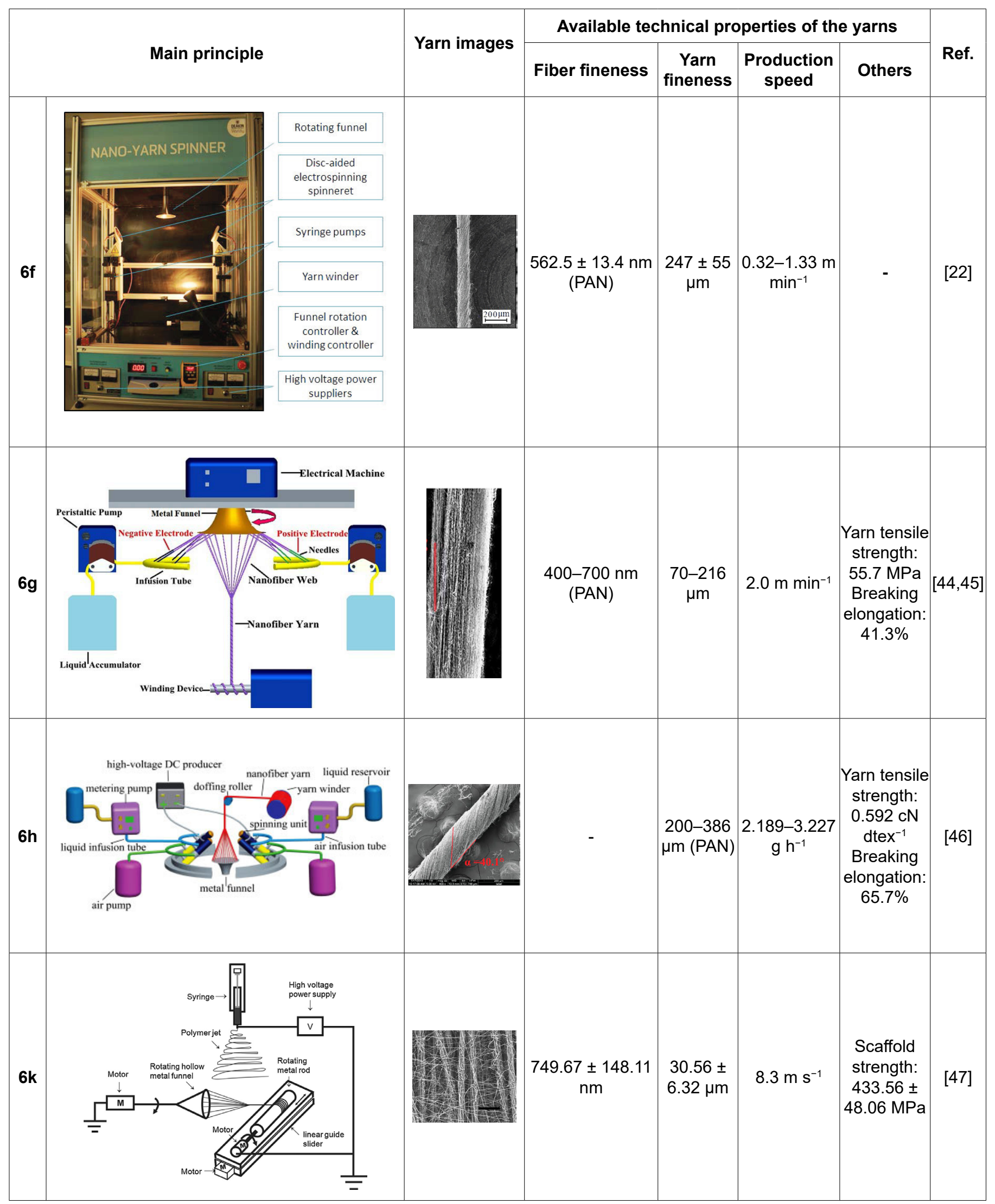




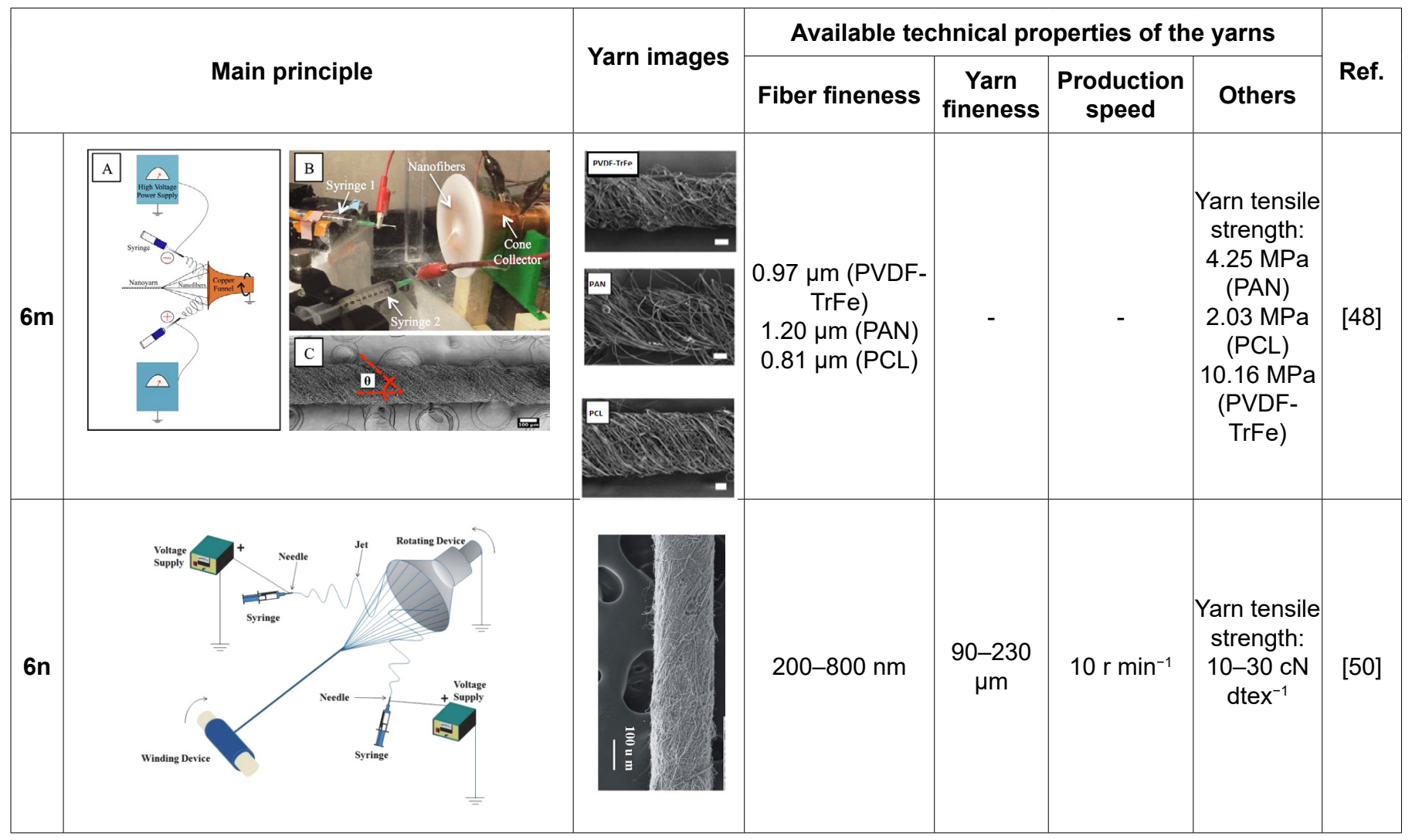

work, hybrid nanoyarns from PVA and PAN polymers were spun. However, the length of yarn was limited in this work.

A method of splitting and twisting has been another approach to transform the nonwoven nanofibers into yarns as proposed by Nakashima et al. [59], gently cutting into a ribbon-shaped electrospun PVDF fibrous membrane using a knife and then twisting it by a spindle. However, similar to some of the methods mentioned earlier, there is no real yarn spinning process here and it is a discontinuous process (Table $7 \mathrm{j}$ ).

A similar approach was also used by Tsai [60]. In this work, oriented fibers were collected on a rotating disc with four aluminum bars. In order to collect the fibers and twist them into a yarn, a special twisting device was designed and staple nanoyarns were produced (Table $7 \mathrm{k}$ ). However, the system is able to produce a limited length of yarn, such as between 10 and $30 \mathrm{~cm}$.

Different from these, core-yarn production by electrospinning is gaining interest; however, the first attempt belongs to Formhals [11], as already mentioned. More recently, both nanofiber yarn production and core-yarn production have been presented by Su et al. [61] using a 20-tex thick filament covered by nanofibers (Table $7 \mathrm{~m})$. However, the yarn tenacity was quite low $(0.26 \mathrm{cN}$ $\mathrm{dtex}^{-1}$ ) if no core filament was used when compared to that for core-spun yarns, which is $3.25 \mathrm{cN} \mathrm{dtex}{ }^{-1}$. Such a concept of collecting fibers as strips onto a revolving collector first and then applying twist to these strips has also been used by Chawla et al. [38], as mentioned in the earlier parts.

More recently, an interesting work was also reported using an AC power supply to produce a fiber group named as "nanofibrous plume" emanating from the virtual collector [62].
The apparatus works without an electrically active collector, and authors indicate that AC process possesses some kind of self-organized counter electrode. In this design, the ends of the nanofibrous plume was attached to a circular brush that, in turn, was attached to rotary tool capable of producing a rotation speed between 5,000 and 20,000 rpm and short lengths of nanofibrous yarns at a predetermined twist density were produced. However, there are no further data for yarn properties, and further work is needed to analyze the full potential of such an approach for a real twisted electrospun yarn production at continuous length (Table $7 n$ ).

Bosworth [63] reports a system collecting fibers as a fibrous ribbon on the edge of a mandrel rotating at $600 \mathrm{rpm}$. Electrospun yarns were fabricated by removing and cutting the fibrous ribbon into $50 \mathrm{~mm}$ lengths and then briefly submerging in distilled water. The strips were then manually twisted, leading to a yarn that has a diameter of approximately $200 \mu \mathrm{m}$ (Table 7p). Same researchers also reported the use of these electrospun yarns for reconstructing damaged tendons [64].

\section{Conclusions}

Development of a yarn spinning system by electrospinning is a challenging task and there is still no system accepted universally. This work shows that there are more than 20 different approaches or methods available. We categorized these into two main groups as (i) systems for production of parallel bundle of fibers and (ii) systems for production of twisted yarns at continuous length. However, some of the available attempts mainly present basic conceptual ideas; therefore, it is difficult to talk about real nanofiber yarn production or nanofiber yarn structures [6-12], while some works present only images 
Table 7. Other methods used for twisted electrospun nanofiber yarn production

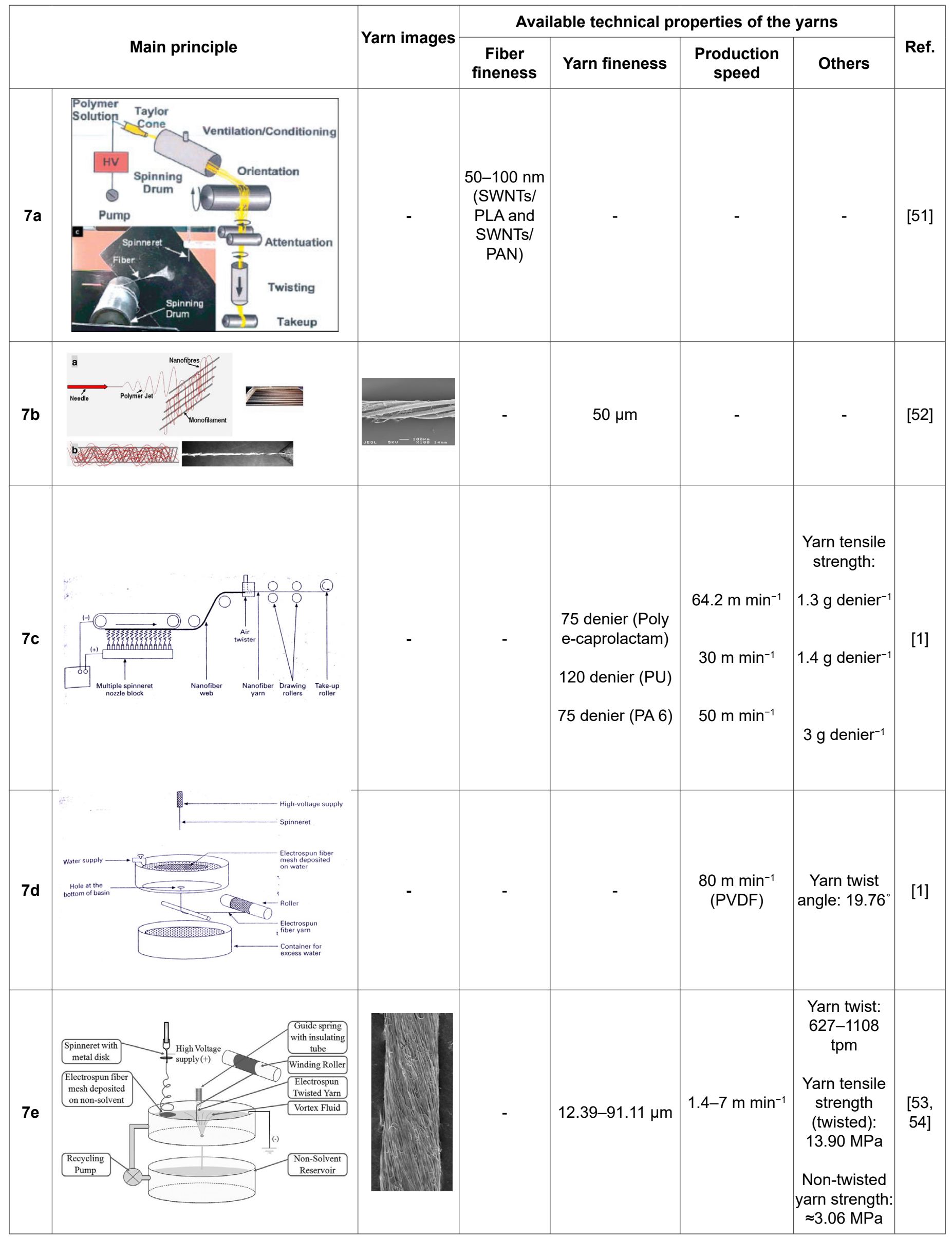




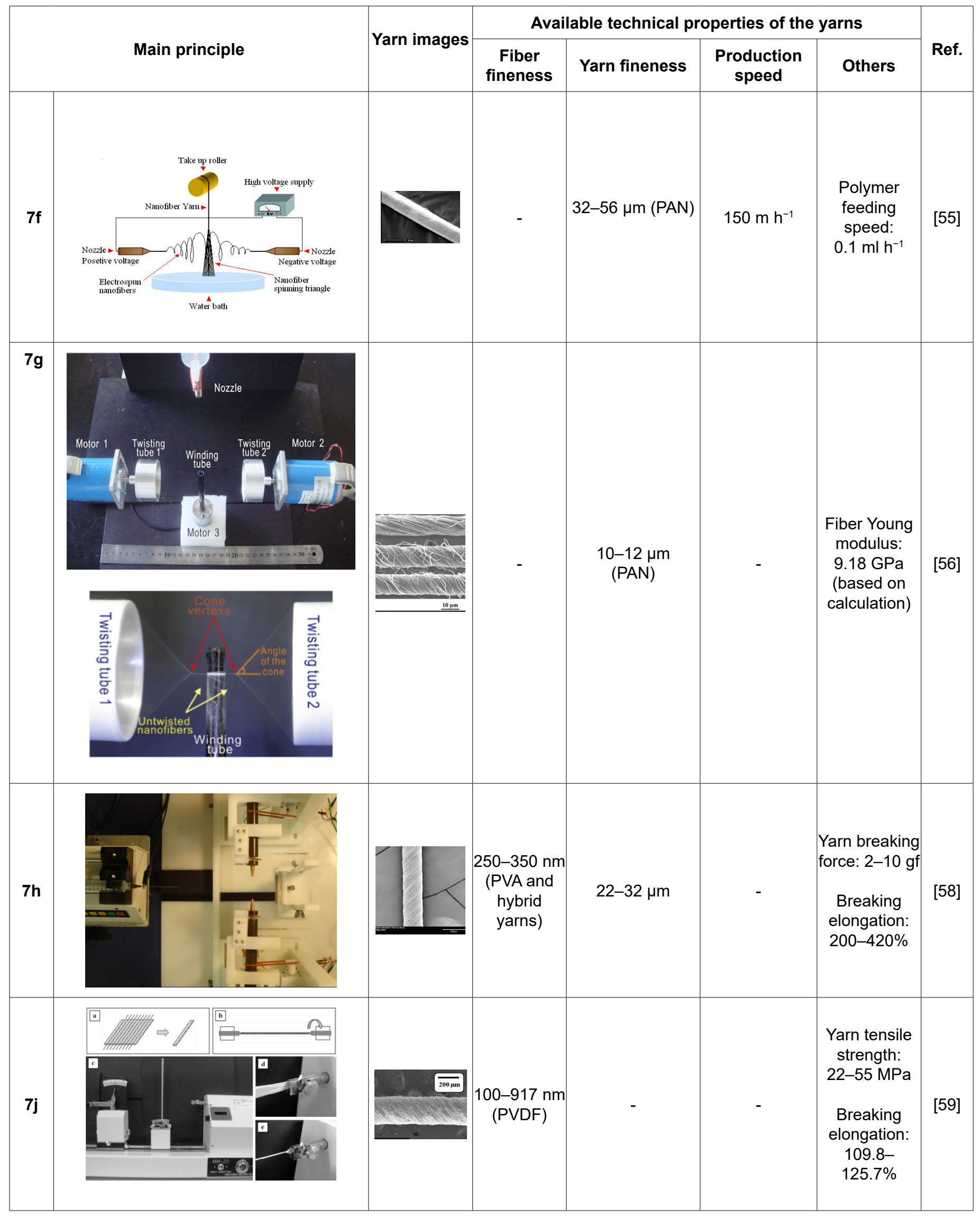




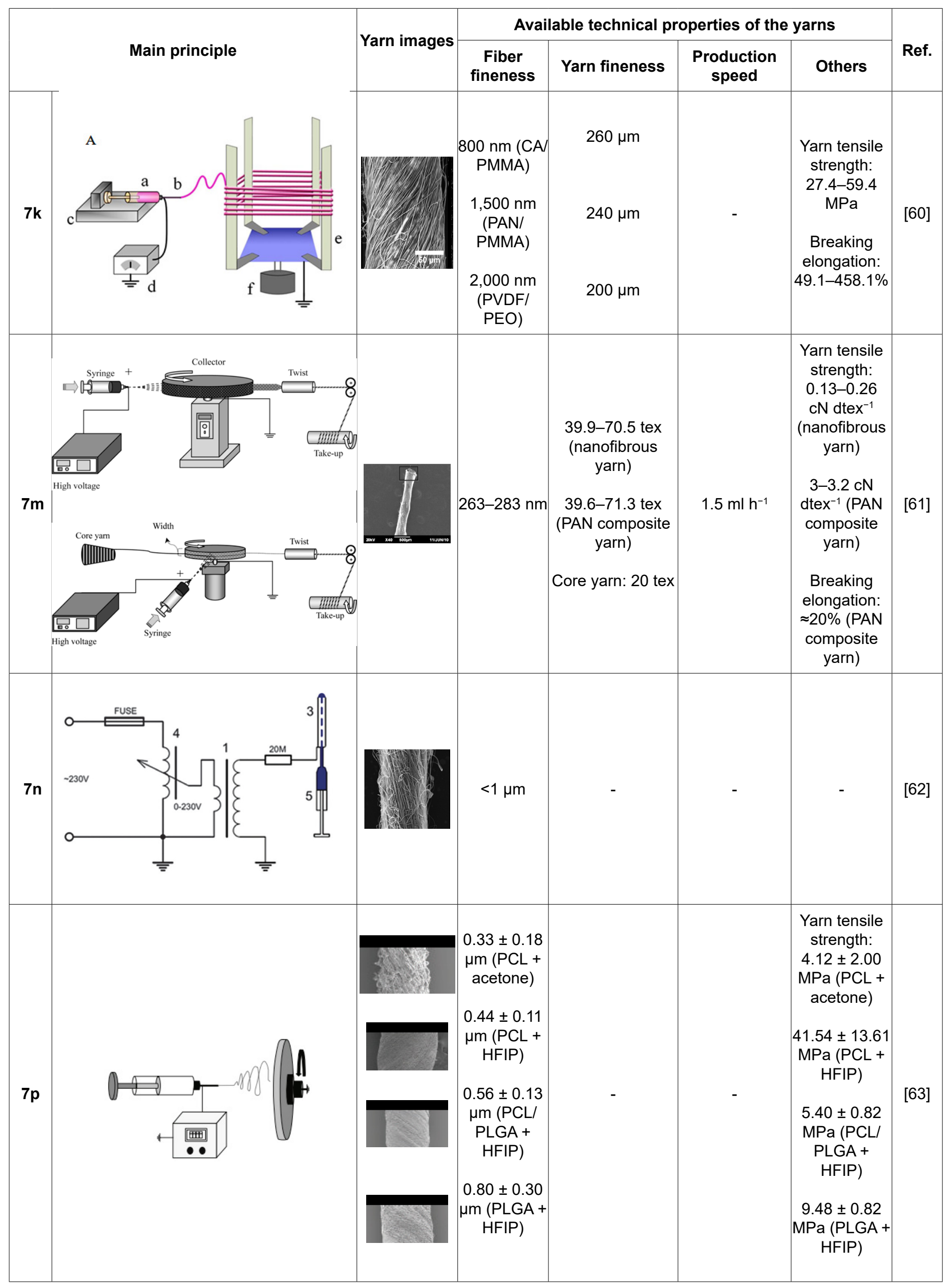


belong to fiber webs or oriented fiber arrays [18, 19, 23]. On the other hand, some studies, although they are valuable attempts for further works, do not present a real yarn spinning system because they mainly produce nanofiber web strips at a certain length first, followed by twisting or plying of these strips at a next step, therefore, being a discontinuous process rather than a real spinning system $[1,32,38,39,59,60,61,63]$. There are also attempts that can produce real twisted nanofiber yarns continuously; however, there is usually no information about the stability of the spinning system or yarn length that can be spun without any break. These results show that currently available systems mainly produce short lengths of yarns with a few exceptions such as the works by Xie, Ali et al., and Shuakat and Lin for successful nanofiber yarn production in bobbin form [22, 25, 40]. Regarding the systems to produce twisted spun yarns, the control of twist level during production is another important task. However, this was usually neglected because the main aim was the development of a successful spinning system. Some other studies provide nominal twist level based on the calculation in conventional textile spinning or yarn surface twist angle [22, 25, 40]. Furthermore, some of the systems, such as Aslı et al. [55], Li et al. [43], Levitt et al. [48], provide very limited data about yarn twisting mechanism, winding system, or winding speed.

Regarding nanofiber yarn properties, a wide range of polymers are used according to the desired application area or for the sake of easy processing in electrospinning. The available works show that there is a high variation in yarn fineness, which ranges from $5 \mu \mathrm{m}$ [33] to 120 denier [1], while some of the reported yarns being rather coarse such as 19 tex [42] or even 70.5 tex [61]. On the other hand, fiber fineness changes between 100 and $1,500 \mathrm{~nm}[16,25]$. Low values for yarn tenacity have been another significant issue that needs to be improved [1, 14, 41, $46,61]$. Yarn production speed is also important in spinning. According to the available data, it significantly depends on the system,for example it is given as $0.32 \mathrm{~m} / \mathrm{min}$ [22], $5 \mathrm{~m} / \mathrm{min}$ [41] or can be as high as $80 \mathrm{~m} / \mathrm{min}$ [1].

This review shows that nanofiber yarn production by a real twisting mechanism for a continuous yarn production at a desired speed has been a real interest and there is still no exact control over nanofiber yarn parameters such as yarn fineness, fiber fineness, or real yarn twist, although some promising approaches are available. The systems developed by Xie [22], Ali et al. [25], and Shuakat and Lin [40] might be good examples at this point. Low production speed and spinning instability seem to be the other main issues to tackle in this field. Consequently, still much work is needed for a successful and fully controllable electrospun nanofiber yarn spinning system accepted universally following the first attempts for electrospun yarn production back in 1930s.

\section{References}

[1] Smit, E., Buttner, U., Sanderson, R. D. (2010). Nanofibers and Nanotechnology in Textiles. (Brown, P. J., Stevens, K. eds.). Woodhead Publishing Limited (Cambridge).

[2] Moon, S., Farris, R. J. (2009). Strong Electrospun Nanometer-Diameter Polyacrylonitrile Carbon Fiber Yarns. Carbon, 47, 2829-2839.
[3] Lotus, A. F. (2009). Synthesis of Semiconducting Ceramic Nanofibers, Development of P-N Junctions and Bandgap Engineering by Electrospinning. Ph.D. Thesis, The University of Akron, Graduate Faculty, Ohio, USA.

[4] Ali, U., Zhou, Y., Wang, X., Lin, Y. (2011). Electrospinning of Continuous Nanofiber Bundles and Twisted Nanofiber Yarns. (Dr. Tong Lin (Ed.). Nanofibers - Production, Properties and Functional Applications, InTech, 153-174.

[5] Wei, L., Qin, X. (2017). Nanofiber Bundles and Nanofiber Yarn Device and Their Mechanical Properties: A Review. Textile Research Journal, Vol. 86 (17), 1885-1898.

[6] Formhals, A. (1938). Artificial Fiber Construction. United States Patent Application 2,109,333.

[7] Formhals, A. (1938). Method and Apparatus for the Production of Fibers. United States Patent Application 2,123,992.

[8] Formhals, A. (1939). Method and Apparatus for Spinning. United States Patent Application 2,160,962.

[9] Formhals, A. (1944). Method and Apparatus for Spinning. United States Patent Application 2,349,950.

[10] Formhals, A. (1934). Process and Apparatus for Preparing Artificial Threads. United States Patent Application 1,975,504.

[11] Formhals, A. (1940). Artificial Thread and Method for Producing Same. United States Patent Application 2,187,306.

[12] Formhals, A. (1943). Production of Artificial Fibers from Fiber Forming Liquids. United States Patent Office Application 2,323,025.

[13] Pan, H., Li, L., Hu, L., Cui, X. (2006). Continuous Aligned Polymer Fibers Produced by a Modified Electrospinning Method. Polymer 47, 4901-4904.

[14] Li, X., Yao, C., Sun, F., Song, T., Li, Y., Pu, Y. (2007). Conjugate Electrospinning of Continuous Nanofiber Yarn of Poly(L-lactide)/Nanotricalcium Phosphate Nanocomposite. Journal of Applied Polymer Science, Vol. 107, 3756-3764.

[15] Teo, W. E., Gopal, R., Ramaseshan, R., Fujihara, K., Ramakrishna S. (2007). A Dynamic Liquid Support System for Continuous Electrospun Yarn Fabrication. Polymer, 48, 3400-3405.

[16] Maheshwari, S., Chang, H. (2009). Assembly of MultiStranded Nanofiber Threads through AC Electrospinning. Advanced Material, Vol. 21, 349-354.

[17] Mondal, A., Borah, R., Mukherjee, A., Basu, S., Jassal, M., Agraw, A. K. (2008). Electrospun Self-Assembled Nanofiber Yarns. Journal of Applied Polymer Science, Vol. 110, 603-607.

[18] Lee, J. R., Jee, S. Y., Kim, H. J., Hong, Y. T., Kim, S., Park, S. J. (2010). Filament Bundle Type Nanofiber and Manufacturing Method. US Patent Application 7,803,460B2.

[19] Kim, H. Y. (2010). Method of Manufacturing a Continuous Filament by Electrospinning. United States Patent Application 7,799,262B1.

[20] Wang, X., Zhang, K., Zhu, M., Yu, H., Zhou, Z., Chen, Y., Hsiao, B. S. (2008). Continuous Polymer Nanofiber Yarns Prepared by Self-bundling Electrospinning Method. Polymer, 49, 2755-2761.

[21] Wang, X., Zhang, K., Zhu, M., Hsiao, B., Chu, B. (2008). Enhanced Mechanical Performance of SelfBundled Electrospun Fiber Yarns via Post-Treatments. Macromolecular Rapid Communications, 29, 826-831. 
[22] Xie, Z. (2013). Polyacrylonitrile Nanofibre Yarn; Electrospinning and Their Post-Drawing Behavior. Ph.D. Thesis, Deakin University, Australia.

[23] Dabirian, F., Ravandi, H. S. A., Hashemi, S. R., Hinestroza, J. P. (2011). Manufacturing of Twisted Continuous PAN Nanofiber Yarn by Electrospinning Process. Fibers and Polymers, Vol. 12 (5), 610-615.

[24] Baura, B. (2015). Investigation of Electrospinning Process Parameters and Studies of Stabilization Kinetics of Polyacrylonitrile-Based Electrospun Carbon Nanofibers. Ph.D. Thesis, University of Oklahoma, Graduate College, Oklahoma, USA.

[25] Ali, U., Zhou, Y., Wang, X., Lin, T. (2012). Direct Electrospinning of Highly Twisted, Continuous Nanofiber Yarns. The Journal of the Textile Institute, 1031, 80-88.

[26] Dabirian, F., Hosseini, Y., Ravandi, H. S. A. (2007). Manipulation of the Electric Field of Electrospinning System to Produce Polyacrylonitrile Nanofiber Yarn. The Journal of the Textile Institute, 98-3, 237-241.

[27] Dabirian, F., Ravandi, H. S. A. (2009). Novel Method for Nanofibre Yarn Production Using Two Differently Charged Nozzles. Fibres \& Textiles in Eastern Europe, Vol. 17, No. 3 (74), 45-47.

[28] Hajiani, F., Jeddi, A. A. A., Gharehaghaji, A. A. (2012). An Investigation on the Effects of Twist on Geometry of the Electrospinning Triangle and Polyamide 66 Nanofiber Yarn Strength. Fibers and Polymers, Vol.13 (2), 244-252.

[29] Maleki, H., Gharehaghaji, A. A., Moroni, L., Dijkstra, P. J. (2013). Influence of the Solvent Type on the Morphology and Mechanical Properties of Electrospun PLLA Yarns. Biofabrication, 5, 035014-035021.

[30] Maleki, H., Gharehaghaji, A. A., Criscenti, G., Criscenti, G., Moroni, L., Dijkstra, P. J. J. (2015). The Influence of Process Parameters on the Properties of Electrospun PLLA Yarns Studied by the Response Surface Methodology. Applied Polymer Science, 132, 41388.

[31] Memarian, F., Latifi, M., Armani-Tehran, M. (2014). Innovative Method for Electrospinning of Continuous TiO2 Nanofiber Yarns: Importance of Auxiliary Polymer and Solvent Selection. Journal of Industrial and Engineering Chemistry, Vol. 20, 1886-1891.

[32] Tian, L., Yan, T., Pan, Z. (2015). Fabrication of Continuous Electrospun Nanofiber Yarns with Direct 3D Processability by Plying and Twisting. Journal of Mater Science, 50, 7137-7148.

[33] Bazbouz, M. B., Stylios, G. K. (2008). Novel Mechanism for Spinning Continuous Twisted Composite Nanofiber Yarns. European Polymer Journal, 44 (1), 1-12.

[34] Bazbouz, M. B., Stylios, G. K. (2009). A New Mechanism for the Electrospinning of Nano Yarns. Journal of Applied Polymer Science, 124, 195-201.

[35] Dabirian, F., Ravandi, H. S. A., Pishevar, A. R., Abuzade, R. A. (2011). A Comparative Study of Jet Formation and Nanofiber Alignment in Electrospinning and Electrocentrifugal Spinning Systems. Journal of Electrostatics, Vol. 69, 540-546.

[36] Wu, S., Qin, X. (2013). Uniaxially Aligned Polyacrylonitrile Nanofiber Yarns Prepared by a Novel Modified Electrospinning Method. Materials Letters, 106, 204-207.
[37] Wu, S., Zhang, Y., Liu, P., Qin, X. (2014). Polyacrylonitrile Nanofiber Yarns and Fabrics Produced Using a Novel Electrospinning Method Combined With Traditional Textile Techniques. Textile Research Journal, Vol. 86 (16), 17161727.

[38] Chawla, S., Naraghi, M., Davoudi, A. (2013). Effect of Twist and Porosity on the Electrical Conductivity of Carbon Nanofiber Yarns. Nanotechnology, 24 (25), 255708255717.

[39] Fennessey, S. F., Farris, R. J. (2004). Fabrication of Aligned and Molecularly Oriented Electrospun Polyacrylonitrile Nanofibers and the Mechanical Behaviour of Their Twisted Yarns. Polymer, 45, 4217-4225.

[40] Shuakat, M. N., Lin, T. (2015). Direct Electrospinning of Nanofibre Yarns Using a Rotating Ring Collector. The Journal of the Textile Institute, 107, 6, 791-799.

[41] Afifi, A. M., Nakano, S., Yamane, H., Kimura, Y. (2010). Electrospinning of Continuous Aligning Yarns with a Funnel Target. Macromolecular Materials and Engineering, 2957, 660-665.

[42] Ravandi, S. A. H., Bayat, T. R., Dabirian, F., Gharehaghaji, A. A., Sajjadi, A. (2015). Characteristics of Yarn and Fabric Made out of Nanofibers. Materials Sciences and Application, 6, 103-110.

[43] Li, N., Hui, Q., Xue, H., Xiong, J. (2012). Electrospun Polyacrylonitrile Nanofiber Yarn Prepared by FunnelShape Collector. Materials Letters, Vol 79, 245-247.

[44] He, J., Qi, K., Zhou, Y., Cui, S. (2014). Multiple Conjugate Electrospinning Method for the Preparation of Continuous Polyacrylonitrile Nanofiber Yarn. Journal of Applied Polymer Science, Vol. 8, 40137-40143.

[45] He, J., Zhou, Y., Qi, K., Wang, L., Li, P., Cui, S. (2013). Continuous Twisted Nanofiber Yarns Fabricated by Double Conjugate Electrospinning. Fibers and Polymers, Vol.14, 11, 1857-1863.

[46] He, J., Qi, K., Zhoua, Y., Cui, S. (2014). Fabrication of Continuous Nanofiber Yarn Using Novel Multi-Nozzle Bubble Electrospinning. Polymer Int., 63, 1288-1294.

[47] Yang, H., Deng, G., Chen, W., Ye, X., Mo, X. (2014). A Novel Electrospun-Aligned Nanoyarn-Reinforced Nanofibrous Scaffold for Tendon Tissue Engineering. Colloids and Surfaces B: Bio Interfaces, 122, 270-276.

[48] Levitt, A. S., Knittel, C. E., Vallett, R., Koerner, M., Dion, G., Schauer, C. L. (2017). Investigation of Nanoyarn Preparation by Modified Electrospinning Set up. Journal of Applied Polymer Science, 134, 44813.

[49] Göktepe, F., Buzol Mülayim, B. (2015). Electrospun Yarn Production by Use of Funnel Collector. 15th AUTEX World Textile Conference, June 10-12, Bucharest, Romania.

[50] Jin, S., Xin, B., Zheng,Y. (2017). Preparation and Characterization of Polysulfone Amide Nanoyarns by the Dynamic Rotating Electrospinning Method. Textile Research Journal, 0(00), 1-11.

[51] Ko, F., Gogotsi, Y., Ali, A., Naquib, N., Ye, H., Yang, G. L., Li, C., Willis, P. (2003). Electrospinning of Continuous Carbon Nanotube Filled Nanofiber Yarns. Adv. Materials, 15 (14), 1161-1165.

[52] Zhou, F., Gong, R., Porat, I. (2010). Nano-Coated Hybrid Yarns Using Electrospinning. Surface \& Coatings Technology, 204, 3459-3463. 
[53] Latifi, M., Yousefzadeh, M., Amani-Tehran, M., Ramaksihna, S. (2011). Continuous Twisted Yarn from Well Aligned Nanofibers by a Water Vortex. Society of Plastics Engineers, Retrieved on May 15, 2016. Web Site: https://www.academia.edu/1456177/Continuous_twisted_ yarn_from_wellaligned_nanofibers_by_a_water_vortex.

[54] Yousefzadeh, M., Latifi, M., Teo, W. E., Armani-Tehran, M., Ramakrishna, S. (2011). Producing Continuous Twisted Yarn from Well-Aligned Nanofibers by Water Vortex. Polymer Eng. Science, 51, 323-329.

[55] Aslı, M. M., Gharehaghaji, A., Johari, M. S. (2010). Study on the Effects of Application of Surface Tension on the Structure of Electrospun Nanofiber Yarn. 7th International Conference TEXSCI, Liberec. Retrieved on May 15, 2017. Web Site: http://wjoe.hebeu.edu.cn/ sup.2010/Nanofibers\%20and\%20Nanotechnologies/4_p_ Gharehgahaji.pdf

[56] Yan, H., Liu, L., Zhang, Z. (2011). Continually Fabricating Staple Yarns with Aligned Electrospun Polyacrylonitrile Nanofibers. Materials Letters, 65, 2419-2421.

[57] Hearle, J. W. S. (1969). In Structural Mechanics of Fibers, Yarn and Fabrics. (Hearle, J. W. S., Grosberg, P., Backer, S., eds.). John Wiley \& Sons, USA.

[58] Bagherzadeh, A. S. (2010). Electrospinning Yarn Formation and Coating. Ph.D. Thesis, Graduate of Faculty of North Carolina State University, North Carolina, US.
[59] Nakashima, R., Watanabe, K., Lee, Y., Kim, B. S., Kim, I. (2013). Mechanical Properties of Poly(vinylidene fluoride) Nanofiber Filaments Prepared by Electrospinning and Twisting. Advances in Polymer Technology, Vol. 32, No. S1, E44-E52.

[60] Tsai, C. C. (2013). Electrospun Nanofiber Yarns for Nanofluidic Applications. Ph.D. Thesis, Graduate School of Clemson University, South Carolina, US.

[61] Su, C., Lai, T., Lu, C., Liu, Y., Wu, S. (2013). Yarn Formation of Nanofibers Prepared Using Electrospinning. Fibers and Polymers, Vol.14, No.4, 542-549.

[62] Pokorny, P., Kostakova, E., Sanetrnik, F., Mikes, P., Chvojka, J., Kalous, T., Bilek, M., Pejchar, K., Valtera, J., Lukas, D. (2014). Effective AC Needleless and Collectorless Electrospinning for Yarn Production. Physical Chemistry Chemical Physics, Vol. 16, 48, 26816-26822.

[63] Bosworth, L. A. (2014). Travelling Along the Clinical Roadmap: Developing Electrospun Scaffolds for Tendon Repair. UK Society for Biomaterials Annual Conference, 2013, Hindawi Publishing: Conference Papers in Materials Science, Vol. 2014.

[64] Bosworth, L. A., Rathbone, S. R., Bradley, R. S., Cartmell, S. H. (2014). Dynamic Loading of Electrospun Yarns Guides Mesenchymal Stem Cells Towards a Tendon Lineage. Journal of the Mechanical Behaviour of Biomedical Materials, 39, 175-183. 